\title{
Mechanosensitive control of mitotic entry
}

Margarida Dantas ${ }^{1,2}$, Andreia Oliveira ${ }^{1}$, Paulo Aguiar $^{1}$, Helder Maiato ${ }^{1,3,4}$, Jorge G. Ferreira $^{1,3,4 *}$

1 - Instituto de Investigação e Inovação em Saúde (i3S), Universidade do Porto, Portugal

2 - BiotechHealth PhD program, Instituto de Ciências Biomédicas Abel Salazar (ICBAS), Porto, Portugal

3 - Departamento de Biomedicina, Faculdade de Medicina do Porto, Portugal

4 - Instituto de Biologia Molecular e Celular (IBMC), Universidade do Porto, Porto, Portugal

* correspondence to:

Jorge G. Ferreira (ORCID: 0000-0001-6802-3696) - jferreir@ibmc.up.pt Address: Rua Alfredo Allen, 4200-135 Porto, Portugal 


\section{Abstract}

As cells prepare to divide, they must ensure that enough space is available to assemble the mitotic machinery without perturbing tissue homeostasis ${ }^{1}$. To do so, cells undergo a series of biochemical reactions regulated by cyclin B1-CDK1 that trigger the reorganization of the actomyosin cytoskeleton ${ }^{2}$ and ensure the coordination of cytoplasmic and nuclear events ${ }^{3}$.

Along with the biochemical events that control mitotic entry, mechanical forces have recently emerged as important players in the regulation of cell cycle events $^{4-6}$. However, the exact link between mechanical forces and the biochemical events that control mitotic progression remains to be established. Here, we identify a mechanical signal on the nucleus that sets the time for nuclear envelope permeabilization and mitotic entry. This signal relies on nuclear unfolding during the G2-M transition, which activates the stretch-sensitive CPLA2 on the nuclear envelope. This activation upregulates actomyosin contractility, determining the spatiotemporal translocation of cyclin B1 in the nucleus. Our data demonstrate how the mechanosensitive behaviour of cyclin B1 ensures timely and efficient mitotic spindle assembly and prevents chromosomal instability.

\section{Main text}

Cell cycle progression is regulated by cyclins and their associated kinases. One such complex, composed of cyclin B1-CDK1, is responsible for regulating entry into mitosis. The biochemical mechanisms that regulate mitotic entry have been extensively studied in the past (for review see ${ }^{7}$ ). For most of the cell cycle, the cyclin B1-CDK1 complex is inactive, due to low cyclin B1 expression levels 
and its mainly cytoplasmic localization ${ }^{8,9}$. As cells approach late G2/prophase of mitosis, cyclin B1 expression increase ${ }^{10}$, leading to phosphorylation and activation of the cyclin B1-CDK1 complex ${ }^{11,12}$. The active cyclin B1/CDK1 complex then stimulates its own nuclear import ${ }^{3}$ through nuclear pore complexes (NPCs), resulting in a stimulation of chromosome condensation ${ }^{14}$ and nuclear envelope permeabilization (NEP) ${ }^{4}$. Consequently, the cyclin B1-CDK1 complex has been proposed to effectively synchronize cytoplasmic and nuclear events ${ }^{3}$, crucial for mitotic entry and efficient spindle assembly.

The influence of mechanical forces on the cell cycle and some of its key regulators has received considerable attention over the last few years ${ }^{1,6,15-19}$. Experiments performed in isolated cells and epithelial layers have demonstrated that mechanical forces can stimulate the G1-S transition $6,15,17,19,20$ by controlling specific transcriptional programs ${ }^{15,17,19}$. This is likely due to forces imposed on the nucleus ${ }^{21,22}$ that induce its flattening ${ }^{19,23,24}$, facilitating the nuclear accumulation of transcription factors $(\mathrm{TFs})^{23,25}$, changing the organization of both chromatin $^{26}$ and the nuclear envelope $(\mathrm{NE})^{27}$ or altering cell contractility ${ }^{24,28}$.

Evidence for mechanical regulation during other cell cycle phases is more limited. Recently, mechanical stretching was proposed to trigger the G2-M transition by activating Piezo $1^{16}$. Moreover, we and others have shown that cell traction forces decrease during the G2-M transition ${ }^{6,20,29}$, to allow mitotic cell rounding and efficient cell division ${ }^{1,29}$. Although these findings highlight the interplay between physical forces and cell proliferation, it remains unknown whether the main biochemical events that occur during the G2-M transition are mechanically regulated. Importantly, whether the spatiotemporal behaviour of the cyclin B1-CDK1 complex is mechanosensitive and contributes to ensure timely 
and efficient cell division remains unknown. Here, we investigate whether and how cyclin B1 responds to physical forces during the G2-M transition. We show that nuclear deformation triggers a contractility-mediated translocation of cyclin B1 to the nucleus, setting the timing of mitotic entry.

We started by investigating how external stimuli regulate the G2-M transition. We seeded RPE-1 cells expressing H2B-GFP/tubulin-RFP in nonadherent, hydrophobic conditions (PLL-g-PEG) and imaged them as they progressed from G2 to mitosis. Under these conditions, cells failed to enter mitosis (Fig. 1a), confirming that the G2-M transition requires contact with external stimuli. Since cell stretching was previously shown to trigger a G2-M transition in monolayer epithelia ${ }^{16}$, we tested whether mechanically stimulating cells in non-adherent conditions was sufficient to stimulate mitotic entry,. Accordingly, upon acute mechanical stimulation, cells re-gained the ability to enter mitosis (Fig. 1a-c; ${ }^{* \star} p<0.001$ ), indicating that mechanical confinement is sufficient to overcome the lack of external stimuli. Under these confinement conditions, cells in late G2 entered mitosis within approximately $2.2 \pm 1.8 \mathrm{~min}$ (mean $\pm s d)$ after stimulation, ruling out that this event was due to increased cyclin B1 transcription ${ }^{16}$. One alternative hypothesis is that mechanical stimulation accelerates mitotic entry by inducing a premature transport of cyclin B1 to the nucleus, as was previously proposed for YAP or MyoD 23,25 . To confirm it this is the case, we monitored the dynamics of nuclear accumulation of endogenous cyclin B1 tagged with Venus in RPE-1 cells, in normal and confined conditions (Fig. 1d, e). We defined time zero as the lowest fluorescence intensity levels of nuclear cyclin B1 and quantified its increase as cyclin B1 translocated to the nucleus, up until NEP. We verified that mechanical stimulation triggered a fast 
nuclear accumulation of cyclin B1 (Fig. 1d-g; ${ }^{* *} p<0.01$ ), with a corresponding faster permeabilization of the NE and mitotic entry (Fig. 1e, $f ;{ }^{* *} p<0.001$ ). This was not due to a rupture of the nucleus, as we could not detect any obvious tears or gaps in the NE when RPE-1 cells expressing Lap2 $\beta$-mRFP were mechanically stimulated (Supplementary Fig. 1a). In addition, we also observed a delay between cyclin B1 accumulation and tubulin translocation to the nucleus (Fig. 1dg). Together, these findings strongly suggest that the nuclear barrier function remains intact after confinement. Instead, as previously described ${ }^{24}$, confinement promoted an unfolding of the NE which could be readily observed (Supplementary Fig. 1a) and resulted in an increase in the distance between neighbouring NPCs (Supplementary Fig. $1 \mathrm{~b}, \mathrm{c} ;{ }^{* \star *} \mathrm{p}<0,001$ ), when compared to unconfined cells.

Next, to investigate if confinement-induced cyclin B1 translocation was dependent on cyclin B1-CDK1 activation ${ }^{3}$, we imaged cells treated with the CDK1 inhibitor RO-3306, with or without mechanical stimulation (Fig. 1h-j). CDK1 inhibition effectively blocked cyclin B1 translocation to the nucleus (Fig. 1h, j), as expected. Interestingly, confinement was sufficient to overcome the inhibition of CDK1 and force translocation of cyclin B1 to the nucleus (Fig. 1h, j; ${ }^{* *} p<0.001$ ). However, these cells failed to enter mitosis, as nuclear envelope permeabilization (NEP) was blocked by CDK1 inhibition ${ }^{31,32}$. This observation further strengthens the idea that mechanical stimulation per se does not affect the barrier function of the nucleus. Next, we tested if confinement-induced cyclin B1 transport required binding to importin $\beta^{33}$ by treating cells with importazole. Treatment with importazole efficiently blocked cyclin B1 nuclear translocation, even in confinement conditions (Fig. 1k and Supplementary Fig. 2), indicating that the 
accelerated translocation of cyclin B1 to the nucleus cannot be explained by an increased diffusive shuttling alone, but by an active process, dependent on importin $\beta$.

Our data strongly suggest that a mechanical signal controls mitotic entry by regulating cyclin B1 nuclear accumulation. Therefore, we sought to identify potential mechanisms involved in the mechanosensitive transport of cyclin B1 to the nucleus. Given that transmission of mechanical signals to the nucleus depends on the linker of cytoskeleton and nucleoskeleton (LINC) complex ${ }^{21}$, we expressed a dominant-negative form of nesprin (DN-KASH) that prevents its binding to SUN proteins and blocks force propagation ${ }^{21}$. Expression of DN-KASH significantly delayed cyclin B1 nuclear accumulation, with a corresponding delay in NEP (280 \pm 71 sec for DN-KASH vs $201 \pm 38$ sec for the control; mean \pm sd; ${ }^{* *} p=0.002$; Fig. 2a-c). Similar delays in cyclin B1 translocation were observed following myosin II inhibition with para-nitro-blebbistatin ( $\mathrm{p}-\mathrm{N}-\mathrm{blebb}$; Fig. $2 \mathrm{~d}$; Supplementary Fig. 3a; ${ }^{* *} \mathrm{p}<0.01$ ) or ML-7 (Supplementary Fig. 3b, c), inhibition of ROCK with Y-27632 (Fig. 2e; Supplementary Fig. 3d; ${ }^{* *} p<0.01$ ), and actin depolymerization with cytochalasin D (CytoD; Fig. 2f; Supplementary Fig. 3e; $\left.{ }^{* * *} \mathrm{p}<0.001\right)$. Conversely, microtubule depolymerization with nocodazole did not affect the timing of cyclin B1 translocation or NEP (Noc; Fig. 2g; Supplementary Fig. 3f; n.s. - not significant). Importantly, mechanical stimulation not only reverted the delay in cyclin B1 accumulation imposed by expression of DN-KASH (Fig. 2a-d; ${ }^{* *} \mathrm{p}<0.001$ ), but also accelerated both cyclin B1 accumulation and NEP in cells where actomyosin contractility was inhibited (Fig. $2 d-h ;{ }^{* *} p<0.01$; $\left.{ }^{* * *} p<0.001\right)$. Overall, these experiments identify actomyosin-dependent force 
transmission to the nucleus as essential to determine the timing of cyclin B1 translocation.

Our results indicate that mechanical stimulation triggers an unfolding of the NE (Supplementary Fig. 1) and an actomyosin-dependent translocation of cyclin B1 into the nucleus (Fig. 2). Next, we tested whether such a mechanism also acted during an unperturbed G2-M transition. To do so, we evaluated the nuclear irregularity index (NII) of interphase and prophase nuclei using fixed-cell analysis. Our results confirm a decrease in NII in prophase cells, when compared to interphase, indicating an unfolding of the NE (Fig. 3a-c; $\left.{ }^{* *} p<0.001\right)$. Such events were previously associated with increased nuclear tension ${ }^{34}$, which trigger the recruitment and activation of the calcium-dependent, nucleoplasmic phospholipase cPLA2 to the NE. Active cPLA2 then stimulates actomyosin contractility ${ }^{24,28}$, possibly leading to NPC opening and increased nucleocytoplasmic shuttling ${ }^{23}$. If indeed prophase nuclei are under increased tension, it is possible that CPLA2 is recruited to the NE at this stage. Accordingly, we found that CPLA2 is recruited to the NE during prophase, similarly to what happens in confined interphase cells ${ }^{24,28}$ (white arrows; Fig. 3d, e), suggestive of increased NE tension and CPLA2 activation at this stage. If CPLA2 is functionally important to facilitate cyclin B1 translocation, inhibiting its activity should result in a delay in cyclin B1 nuclear accumulation. Indeed, inhibition of cPLA2 activity with AAOCF3 led to a significant decrease in cyclin B1 nucleoplasmic shuttling (Fig. $\left.3 f, h, i ;{ }^{* *} p<0.01 ;{ }^{* *} p<0.001\right)$. Similarly, acutely interfering with internal $\mathrm{Ca}^{2+}$ release, known to trigger cPLA2 translocation and activation ${ }^{34}$, using BAPTA-AM +2APB also decreased cyclin B1 nuclear translocation (Fig. 3g-i; Supplementary Fig. $\left.4 a ;{ }^{* * *} \mathrm{p}<0.001\right)$, as expected. Remarkably, confinement was able to 
stimulate cyclin B1 translocation, even when cPLA2 activity or calcium release were inhibited (Fig. 3f-i). This likely occurs due to confinement-induced unfolding of the NE (Supplementary Fig. 1), that is sufficient to bypass the pharmacological inhibition of contractility and still induce an increase in NPC distance (Supplementary Fig. 4b, c). Together, these observations support the mechanosensitive nature of cyclin B1 nuclear accumulation.

Here, we propose a working model for the mechanical regulation of mitotic entry based on nuclear tension, that activates CPLA2 to determine the dynamics of cyclin B1 nuclear accumulation. One prediction of our model is that interfering with nuclear tension should alter the dynamics of cyclin B1 nucleoplasmic shuttling. To confirm this hypothesis, we seeded cells in a soft hydrogel $(5 \mathrm{kPa})$ or in a rigid glass, inducing low or high nuclear tension, respectively. As predicted, cells on glass were more efficient in cyclin B1 nuclear shuttling, than cells on a soft gel (Fig. $\left.4 a, b ;{ }^{* \star *} p<0.001\right)$. In addition, the timing from cyclin B1 nuclear entry and NEP was reduced for cells in glass, indicating that increased tension facilitates mitotic entry (Fig. $\left.4 c ;{ }^{* * *} p<0.001\right)$.

Nuclear translocation of cyclin B1 sets the time for the G2-M transition ${ }^{35}$. This is essential for preventing untimely mitotic entry, which results in chromosome segregation errors ${ }^{36}$. Similarly, confining cells throughout mitosis also contributes to the occurrence of segregation errors ${ }^{1,37}$. Whether a short confinement during the G2-M transition, which is sufficient to induce premature cyclin B1 translocation and NEP, can result in chromosome segregation errors remains unknown. To test this, we subjected cells in prophase to a short confinement, which was released shortly after NEP (Fig. 5a). This approach should induce mitotic entry, while still providing enough volume for the spindle to 
assemble unconstrained ${ }^{1}$. Cells were then allowed to progress through mitosis unperturbed so that we could determine mitotic timings, as well as the rate of chromosome missegregation. Notably, a significant proportion of cells that were subjected to short confinement entered mitosis with incomplete centrosome separation (Fig. 5a-d), which we and others have shown ${ }^{29,38}$ can increase the frequency of mitotic errors. Detailed analysis of centrosome behaviour during the early stages of spindle assembly under short confinement also revealed that centrosome separation and positioning still depended on kinesin-5 and dynein activities. Accordingly, mechanical confinement could not rescue the monopolar spindles generated by STLC treatment (Supplementary Fig. 5a, b) or the centrosome positioning defects induced by DHC RNAi (Supplementary. Fig. 5ce). As a result, this acute confinement resulted in increased chromosome segregation errors (Fig. 5e; ${ }^{*} \mathrm{p}<0.05$ ) and a slight mitotic delay (Fig. 5e; $24 \pm 7 \mathrm{~min}$ for controls vs. $36 \pm 20$ min for confined cells; $\left.{ }^{*} p<0.05\right)$, when compared to unconfined cells. It is plausible that these errors arise from a confinementinduced acceleration of NEP, preventing cells from properly organizing a mitotic spindle. To test this, we decided to promote cyclin B1 nuclear translocation by permeabilizing the NE with laser microsurgery, therefore anticipating mitotic entry (Fig. 5g). Using this approach, we triggered immediate mitotic entry, which was sufficient to increase chromosome missegregation events (white arrowhead, Fig. $5 \mathrm{~g}, \mathrm{~h} ;{ }^{* \star} \mathrm{p}=0.02$ ) and induce a mitotic delay (Fig. 5i). Together, these experiments demonstrate that untimely mitotic entry through acute mechanical confinement during the G2-M transition, can have deleterious downstream consequences for chromosome segregation. 
The biochemical regulation of the G2-M transition has been studied extensively ${ }^{3,8-12}$. However, how mechanical forces affect this essential step of the cell cycle was unknown. Here, we propose a nongenetic, mechanical pathway based on nuclear tension that acts during the G2-M transition, impacting cyclin B1 translocation and setting the time for NEP and accurate cell division. In agreement with this model, we observed an increased nuclear unfolding in prophase cells (Fig. 3a-c; Supplementary Figs. 1 and 4), similarly to previous observations in $\mathrm{G} 2$ cells $^{24}$. In addition, cPLA2 recruitment to the NE was also increased (Fig. 3d, e). These observations are indicative of cPLA2 activation $24,28,34$ and likely reflect an increased tension on the nucleus during the G2-M transition. Once activated, cPLA2 triggers actomyosin contractility ${ }^{24,28}$, which leads to nuclear deformation ${ }^{19}$. In the context of the G2-M transition, this increased contractility could be sufficient to deform the nucleus and NPCs ${ }^{23,25}$, leading to faster cyclin B1 transport across the NE. In agreement, we were able to rescue cyclin B1 shuttling to the nucleus by mechanical stimulation, even in the absence of cPLA2 activity or actomyosin contractility. Overall, this mechanism would ultimately set the time of NEP and ensure timely and accurate cell division. Establishing mechanical forces as a determinant for cyclin B1 nuclear translocation and mitotic entry raises the interesting possibility that the nucleus might act as a sensor ${ }^{24,28}$ for external forces, regulating cell cycle progression and cell division to control tissue growth and avoid over-proliferation. 


\section{Online Methods}

\section{$\underline{\text { Cell lines }}$}

Cell lines used were cultured in DMEM (Life Technologies) supplemented with $10 \%$ FBS (fetal bovine serum - Life Technologies) and kept in culture in a $3^{\circ} \mathrm{C}$ humidified incubator with 5\% CO 2 . RPE-1 parental and RPE-1 H2B-GFP and tubulin-mRFP were already available in our lab. RPE-1 endogenous cyclinB1Venus cell line was a gift from Jonathon Pines. RPE-1 cyclinB1-Venus/tubulinmRFP cell line was generated in our lab by transduction with lentiviral vectors containing tubulin-mRFP (Addgene). HEK293T cells at a 50-70\% confluence were co-transfected with lentiviral packaging vectors $(16.6 \mu \mathrm{g}$ of Pax2, $5.6 \mu \mathrm{g}$ of pMD2 and $22.3 \mu \mathrm{g}$ of LV-tubulin-mRFP), using 30 $\mu \mathrm{g}$ of Lipofectamin 2000 (Life Technologies). Approximately 4-5 days after the transduction, the viruscontaining supernatant was collected, filtered, and stored at $-80^{\circ} \mathrm{C}$. RPE-1 cyclinB1-Venus cells were infected with virus particles together with polybrene (1:1000) in standard culture media for $24 \mathrm{~h}$. Approximately $2-3$ days after the infection, the cells expressing tubulin were isolated by fluorescence activated cell sorting (FACS; FACS Aria II).

\section{Drug treatments}

CDK1 inhibitor (RO-3306) was used at a concentration of $9 \mu \mathrm{M}$ for $16 \mathrm{~h}$. Importazole was added to the cells at a final concentration of $40 \mu \mathrm{M} 2 \mathrm{~h}$ before the experiment, ROCK inhibitor ( $\mathrm{Y}$-27632) was used at $5 \mu \mathrm{M}$ for $30 \mathrm{~min}$ (Sigma Aldrich). To interfere with cPLA2 activity, AACOCF3 was used at $20 \mu \mathrm{M}$ (TOCRIS) for $30 \mathrm{~min}$. To block the release of calcium ions from internal cellular stores, BAPTA-AM and 2APB (Abcam) were used at $10 \mu \mathrm{M}$ for 15-30 min. Myosin 
activity was perturbed using p-nitro-blebbistatin at $50 \mu \mathrm{M}$ for $30 \mathrm{~min}$ (MotorPharma). MLCK activity was blocked using ML-7 at $50 \mu \mathrm{M}$ for $30 \mathrm{~min}$. To interfere with the actin cytoskeleton, we used cytochalasin D at $0.5 \mu \mathrm{M}$ (TOCRIS) for $30 \mathrm{~min}$. To perturb microtubules, nocodazole was used at $3.3 \mu \mathrm{M}$ for $30 \mathrm{~min}$. Control cells were treated with either DMSO (Sigma) or transfected with Lipofectamin 2000 (Invitrogen), as explained in the text.

\section{$\underline{\text { Transfections }}$}

Cells were transfected with the plasmid encoding the DN-KASH mutant using Lipofectamin 2000 (Life Technologies). Specifically, $5 \mu \mathrm{L}$ of Lipofectamin 2000 and $0.5 \mu \mathrm{g}$ of DN-KASH plasmid were diluted separated and incubated in OPTIMEM (Alfagene) for $30 \mathrm{~min}$. The mixture was then added to confluent cells cultured and incubated for $6 \mathrm{~h}$ in reduced serum medium (DMEM with 5\% FBS). Cells were analyzed $48 \mathrm{~h}$ after transfection.

For the DHC RNAi experiment, cells were transfected with small interfering RNAs (siRNAs) using Lipofectamin RNAi Max (Life Technologies). Specifically, $5 \mu \mathrm{L}$ of Lipofectamin and $20 \mathrm{nM}$ of each siRNA were diluted separated and incubated in OPTIMEM (Alfagene) for $30 \mathrm{~min}$. The mixture was then added to confluent cells cultured and incubated for $6 \mathrm{~h}$ in reduced serum medium (DMEM with 5\% FBS). Commercial ON-TARGETplus SMARTpool siRNAis (Dharmacon) were used. Cells were analyzed $72 \mathrm{~h}$ after transfection and protein depletion efficiency verified by immunoblotting.

\section{Time-lapse microscopy}


Around 12-24 $\mathrm{h}$ before the experiments $1.5 \times 10^{5}$ cells were seeded on fluorodishes (WPI) coated with FBN (25 $\mu \mathrm{g} / \mathrm{mL}$; F1141; Sigma). Shortly before each experiment, DMEM 10\%FBS medium was changed to Leibovitz's-L15 medium (Life Technologies), supplemented with 10\% FBS and AntibioticAntimycotic 100X (AAS; Life Technologies). Live cell imaging experiments were performed using temperature-controlled Nikon TE2000 microscopes equipped with a modified Yokogawa CSU-X1 spinning-disk head (Yokogawa Electric), an electro multiplying iXon+ DU-897 EM-CCD camera (Andor) and a filter wheel. Three laser lines were used to excite $488 \mathrm{~nm}, 561 \mathrm{~nm}$ and $647 \mathrm{~nm}$ and all the experiments were done with oil immersion 60x 1.4NA Plan-Apo DIC (Nikon). Image acquisition was controlled by NIS Elements AR software. Images with 1721 z-stacks (0.5 $\mu \mathrm{m}$ step) were collected with a $20 \mathrm{sec}$ interval.

\section{Western Blotting}

Cell extracts were collected after trypsinization and centrifuged at 1,200 rpm for $5 \mathrm{~min}$, washed and resuspended in 30-50 $\mu \mathrm{L}$ of lysis buffer (20nM HEPES/KOH, pH 7.9; $1 \mathrm{mM}$ EDTA pH8; 150mM NaCl; 0.5\% NP40; 10\% glycerol, 1:50 protease inhibitor; 1:100 Phenylmethylsulfonul fluoride). The cells were then flash frozen in liquid nitrogen and kept on ice for $30 \mathrm{~min}$. After centrifugation at 14,000 rpm for $8 \min$ at $4^{\circ} \mathrm{C}$, the supernatant was collected, and protein concentration determined using the Bradford protein assay (Bio-Rad). The proteins were run on a $10 \%$ SDS-PAGE gel (50 $\mu \mathrm{g} /$ lane $)$ and transferred using a wet blot apparatus for $1.5 \mathrm{~h}$ at $70 \mathrm{~V}$, with constant amperage. Later, the membranes were blocked with 5\% milk in Tris Buffered Saline (TBS) with 0.1\% Tween-20 (TBS-T) for $1 \mathrm{~h}$ at room temperature. The primary antibodies used were anti-dynein (1:250, Thermo 
Fisher Scientific) and anti-vinculin (1:1000, Bio-Rad). All primary antibodies were incubated overnight at $4^{\circ} \mathrm{C}$ with shaking. After three washes in TBS-T, the membranes were incubated with the secondary antibody for $1 \mathrm{~h}$ at room temperature. The secondary antibodies used were anti-rabbit-HRP at 1:5000. After several washes with TBS-T, the detection was performed with Clarity Western ECL Substrate (Bio-Rad).

\section{$\underline{\text { Cell confinement setup }}$}

For dynamic confinement experiments, we adapted a cell confiner as previously described $^{39}$, using a custom-designed polydimethylsiloxane (PDMS, RTV615, GE) layout to fit a $35 \mathrm{~mm}$ fluorodish. A suction cup was custom-made with a 10/1 mixture (w/w PDMS A/crosslinker B), baked on an $80^{\circ} \mathrm{C}$ hot plate for $1 \mathrm{~h}$ and left to dry over-night before unmolding. The confinement slide was polymerized on $10 \mathrm{~mm}$ round coverslips. These round coverslips were first treated with air plasma for 2 min (Zepto system, Diener Electronics) and incubated with a $0.3 \%$ BindSilane (Sigma M6514)/5\% acetic acid solution in ethanol. Then, the coverslips were rinsed with ethanol and left to dry. A gel with approximately $15 \mathrm{kPa}$ stiffness was prepared using an acrylamide (Bio-Rad)/bisacrylamide (Bio-Rad) mix. The mixture was added to the coverslips and allowed to polymerize. After polymerization, gels were hydrated with PBS and incubated with cell culture medium for at least $30 \mathrm{~min}$. The confinement slide was then attached to the PDMS suction cup described above and connected to a vacuum generator apparatus (Elveflow).

For static confinement experiments, we used a commercially available 6-well confinement device (4DCell) with custom designed confinement slides. The 
confinement slide was polymerized in PDMS on a round $10 \mathrm{~mm}$ standard microscope coverslip and designed with a regular holes array (diameter $449 \mu \mathrm{m}$, $1 \mathrm{~mm}$ spacing). Briefly, after activating the coverslip in a plasma chamber (Diener Electronics, Germany) for 2 min, a PDMS drop was pressed on the coverslip to obtain a thin layer. After baking at $95^{\circ} \mathrm{C}$ on a hot plate for $15 \mathrm{~min}$, excess PDMS was removed. Isopropanol was used to peel off the glass slide with the PDMS pillars and to remove the excess of PDMS. Microfabricated coverslips with confining pillars (8 $\mu \mathrm{m}$ height) were then attached to PDMS spacers that were stuck on a 6-well plate lid (4DCell).

\section{$\underline{\text { CH-STED super-resolution microscopy }}$}

For CH-STED microscopy, cells were grown as described above. Parental RPE1 cells were seeded in the day before the experiment in coverslips coated with FBN. After fixation with $4 \%$ paraformaldehyde in cytoskeleton buffer the cells were extracted in PBS with 0.5\% Triton-X100 (Sigma- Aldrich). The coverslips were incubated with the primary antibodies (rabbit anti-TPR, 1:100, NB100-2867; and mouse anti-NUPs, 1:100, Abcam 24609) in blocking solution overnight at $4^{\circ}$ C. After washing with PBS-0.1\%Tríton-X, the coverslips were incubated with the secondary antibodies (Abberior anti-rabbit STAR 580, 1:100, and STAR and Abberior anti-mouse STAR 635P, 1:100) at room temperature for $1 \mathrm{~h}$. Later, coverslips were washed in PBS with $0.1 \%$ Triton-X100 and sealed on a glass slide using mounting medium (20nM Tris $\mathrm{pH} 8,0.5 \mathrm{~N}$-propyl gallate, 90\% glycerol).

The images were acquired with an Abberior Instruments "Expert Line" gatedSTED coupled to a Nikon Ti microscope. For all the acquisitions, an oil-immersion 
60x 1.4NA Plan-Apo objective (Nikon, Lambda Series) and pinhole size of 0.8 Airy units were used. The $\mathrm{CH}$-STED technique creates an orthogonal direction on the STED parametric space that enables the independent tuning of both resolution and contrast using only one depletion beam in a standard STED setup (circular polarization based).

\section{$\underline{\text { Immunofluorescence }}$}

For the immunofluorescence experiments, cells were grown as previously described. RPE-1 parental cells were seeded in the day before the experiment in coverslips coated with FBN. After fixation with 4\% Paraformaldehyde in cytoskeleton buffer $\left(274 \mathrm{mM} \mathrm{NaCl}, 2.2 \mathrm{mM} \mathrm{Na}_{2} \mathrm{HPO}_{4}, 10 \mathrm{mM} \mathrm{KCL}, 0.8 \mathrm{mM}\right.$ $\mathrm{KH}_{2} \mathrm{PO}_{4}, 4 \mathrm{mM}$ EDTA, 4mM MgCl $2,10 \mathrm{mM}$ Glucose, $\mathrm{pH}$ 6.1), cells were extracted with PBS-0.5\% Triton-X100 (Sigma- Aldrich) following three washes (5 min each) with PBS- $0.1 \%$ Triton-X100 and a 30 min incubation in blocking solution (10\% FBS in 10\% Triton-X100 in PBS). The coverslips were incubated with the primary antibodies (rabbit anti-cPLA2, 1:100, Cell Signalling; mouse anti-LaminA/C, 1:500, ABCAM; rat anti-tyrosinated $\alpha$-tubulin 1:500, Bio-Rad) in blocking solution for 1 h at room temperature (RT). After washing with PBS-0.1\%Tríton-X for 5 min, the coverslips were incubated with the secondary antibodies (Alexa Fluor 488, 568 and 647, 1:2000; Invitrogen) at RT for 1h. Later, coverslips were washed, 3x, in PBS with $0.1 \%$ Triton-X100 and $1 \mathrm{x}$ with PBS. Images were acquired using an Axiolmager z1 (63x, Plan oil differential interference contract objective lens, 1.4 NA; from Carl Zeiss), coupled with a CCD camera (ORCA-R2; Hamamatsu Photonics) and the Zen software (Carl Zeiss). 


\section{$\underline{\text { Quantitative image analysis }}$}

For the quantifications of cyclin B1 levels, images were analysed using ImageJ. A small square region of interest (ROI) was defined, and cyclin B1 fluorescence intensity measured, throughout time in the cell nucleus. The same ROI was used to measure the background outside the cell area. All fluorescence intensity values were then background corrected and the values were normalized to the lowest nuclear cyclin B1 level. Time zero was defined as the lowest nuclear cyclin B1 intensity inside the nucleus.

For quantification of cPLA2 fluorescence intensity on the NE, images were analysed using ImageJ. A defined ROI was used to measure the fluorescence intensity values in 5 different regions outside the cells, which was then used to calculate the average background levels. Afterwards, an equivalent ROI was used to measure fluorescence intensity in the nucleoplasm and at the NE. Images were background-subtracted and then area normalized. cPLA2 enrichment at the NE was calculated by obtaining ratio between fluorescence in the NE and in nucleoplasm.

Nuclear irregularity index (NII) was used to estimate the overall folding of the nucleus. To do so, we first obtained 2D images of the nucleus using an anti-Lamin A/C antibody. These images were processed to obtain nuclear area and convex using ImageJ. Nuclear solidity was then calculated as area/convex area. Nuclear Irregularity Index was defined as 1-nuclear solidity.

\section{MATLAB custom algorithm for nuclear pore analysis}

A computational algorithm was developed in MATLAB (The MathWorks Inc, USA; v2018b) to quantify compression-induced topological changes in the nuclear 
pores, within the nuclear membrane. For the analyses done in this study, we used a method focused on estimating changes on the average inter-distance between TPR and a mixture of proteins that compose the nuclear pore complex (NPC), respectively tagged with Abberior anti-rabbit STAR 580 and anti-mouse STAR 635P. Spatial periodicity on either staining (TPR and NPC) was estimated through a spatial Fast-Fourier Transform (FFT) operation on membrane crosssection images. As an alternative, and as validation, intensity profile autocorrelation was also used to assess the spatial periodicity. Only segments with low curvatures were used with both approaches, and the "Straighten" tool ${ }^{41}$, was used prior to the FFT/autocorrelation operations.

\section{MATLAB custom algorithm for centrosome tracking}

To perform a detailed quantitative analyse of the centrosome positioning and movement, we used a custom-designed MATLAB (The MathWorks Inc, USA; v2018b) script that was previously described ${ }^{29}$. The algorithm used a specific workflow designed for centrosome tracking in a 3D space having in consideration a pixel size of $0.176 \mu \mathrm{m}$ and a z-step of $0.5 \mu \mathrm{m}$. The nucleus shape was reconstructed using $\mathrm{H} 2 \mathrm{~B}-\mathrm{GFP}$ as marker. Using this tool, we were able to correlate the angle between the centrosomes and the nucleus, at the moment of NEP.

\section{Laser microsurgery}

Laser microsurgery was performed with a doubled-frequency laser (FQ-500-532; Elforlight) coupled with an inverted microscope (TE2000U; Nikon), using a 100x 1.4NA, plan-apochromatic DIC objective lens and equipped with an iXonEM + 
EM-CD camera (Andor Technology). To induce a break on the NE, we used 8 consecutive pulses, with a pulse energy of 3-5 $\mu \mathrm{J}$ and an interval of around 10ns.

\section{$\underline{\text { Statistical analysis }}$}

Three to six independent experiments were used for statistical analysis. Knockdown efficiency was assessed by immunoblots quantification. When data are represented as box-whisker plots, the box size represents $75 \%$ of the population and the line inside the box represents the median of the sample. The size of the bars (whiskers) represents the maximum (in the upper quartile) and the minimum (in the lower quartile) values. Statistical analysis for multiple group comparison was performed using a parametric one-way analysis of variance (ANOVA) when the samples had a normal distribution. Otherwise, multiple group comparison was done using a nonparametric ANOVA (Kruskal-Wallis). Multiple comparisons were analyzed using either post-hoc Student-Newman-Keuls (parametric) or Dunn's (nonparametric) tests. When only two experimental groups were compared, we used either a parametric $t$ test or a nonparametric Mann-Whitney test. Distribution normalities were assessed using the Kolmogorov-Smirnov test. No power calculations were used. All statistical analyses were performed using SigmaStat 3.5 (Systat Software, Inc.).

\section{Acknowledgments:}

This work was funded by Portuguese funds through FCT-Fundação para a Ciência e a Tecnologia/Ministério da Ciência, Tecnologia e Ensino Superior in the framework of the project PTDC/BIA-CEL/6740/2020. M.D. is supported by grant PD/BD/135548/2018 from the BiotechHealth FCT-funded PhD program. 
Work in the Maiato lab is funded by the European Research Council (ERC) consolidator grant CODECHECK, under the European Union's Horizon 2020 research and innovation programme (grant agreement 681443), Fundação para a Ciência e a Tecnologia of Portugal (PTDC/MED-ONC/3479/2020), and the NORTE-01-0145-FEDER-000051 project supported by NORTE 2020 under the PORTUGAL 2020 Partnership Agreement through the European Regional Development Fund. The authors would like to thank Jonathon Pines for the gift of the RPE-1 cyclin B1-Venus cell line. The authors also thank members of the Ferreira and Maiato labs for critical reading of the manuscript.

\section{Author contributions:}

M.D. performed experimental work, analyzed data, prepared figures and wrote the manuscript. J.G.F. provided the conceptual framework, analyzed data, prepared figures, and wrote the manuscript. H.M. provided access to essential equipment, reviewed, and edited the manuscript. A.O. and P.A developed MATLAB computational tools, reviewed, and edited the manuscript.

\section{Figure legends}

\section{Figure 1}

(a) RPE-1 cell expressing H2B-GFP and tubulin-mRFP seeded on PLL-g-PEG.

(b) RPE-1 cell expressing H2B-GFP/tubulin-mRFP dividing on PLL-g-PEG, under confinement. Time is in min:sec and time zero corresponds to NEP. Images were acquired with a $20 \mathrm{sec}$ interval. Scale bar corresponds to $10 \mu \mathrm{m}$. (c) Comparison between the percentage of cells that enter mitosis when seeded in non-adherent conditions, with $(n=10)$ and without confinement $\left(n=6 ;{ }^{* * *} p<0.001\right)$. RPE-1 cell 
expressing cyclinB1-Venus/tubulin-mRFP dividing on an FBN-coated substrate without $(\mathbf{d} ; n=26)$ or with $(\mathbf{e} ; n=19)$ confinement. Time is in min:sec and time zero corresponds to NEP. Images were acquired with a $20 \mathrm{sec}$ interval. Scale bar corresponds to $10 \mu \mathrm{m}$. (f) Time lag between cyclin B1 and tubulin nuclear translocation with (green) and without (black) confinement. (g) Normalized cyclin B1 fluorescence accumulation, inside the nucleus, over time in control nonconfined cells (black) and confined (green) cells. Time zero corresponds to the lowest intensity value measured inside the cell nucleus, before NEP. (h) RPE-1 cells expressing cyclinB1-Venus/tubulin-mRFP dividing on a FBN coated substrate, treated with DMSO (top panel; $n=15$ ), a CDK1 inhibitor (middle panel, CDK1i; RO-3306; $n=23$ ) or with CDK1i and confinement (bottom panel; $n=17$ ). Time is in min:sec and time zero corresponds to NEP. Images were acquired with a $20 \mathrm{sec}$ interval. Scale bar corresponds to $10 \mu \mathrm{m}$. (i) Normalized cyclin B1 fluorescence intensity in the nucleus over time in control (DMSO), non-confined cells (black) and DMSO-treated, confined cells (green). Time zero corresponds to the lowest intensity value inside the cell nucleus. (j) Normalized cyclin B1 fluorescence intensity inside the nucleus over time, in CDK1i, non-confined cells (black) and CDK1i, confined cells (green). Time zero corresponds to the lowest intensity value measured inside the cell nucleus, before NEP. (k) Normalized cyclin B1 fluorescence accumulation inside the nucleus over time, in importazoletreated, non-confined cells (black; $n=27$ ) and importazole-treated, confined cells (green; $n=18$ ). Time zero corresponds to the lowest intensity value inside the cell nucleus.

\section{Figure 2}


(a) RPE-1 cell expressing cyclinB1-Venus/tubulin-mRFP dividing on a FBN coated substrate and expressing a DN-KASH mutant dividing without (top panel; $\mathrm{n}=17$ ) or with (bottom panel; $\mathrm{n}=17$ ) confinement. Time is in min:sec and time zero corresponds to NEP. Images were acquired with a $20 \mathrm{sec}$ interval. Scale bar corresponds to $10 \mu \mathrm{m}$. (b) Normalized cyclin B1 fluorescence accumulation, inside the nucleus over time, in control non-confined cells expressing the DN$\mathrm{KASH}$ mutant (black) and confined cells expressing the DN-KASH mutant (green). Time zero corresponds to the lowest intensity value measured inside the cell nucleus, before NEP. (c) Time lag between cyclin B1 and tubulin nuclear translocation in control cells (Lipofectamine, black), non-confined cells expressing the DN-KASH mutant (green) and confined cells expressing the DNKASH mutant (magenta). Normalized cyclin B1 fluorescence intensity inside the nucleus over time in non-confined cells treated with p-nitro-blebbistatin (d; Myosin inhibitor; $n=12), Y-27632$ (e; ROCK inhibitor; $n=21)$, cytochalasin D (f; actin inhibitor; $n=26$ ) and nocodazole ( $\mathbf{g}$; microtubule depolymerizer; $n=20$ ) in black and confined cells with the corresponding treatment (green; $n=17$ for $p$ nitro-blebbistatin; $n=19$ for $Y-27632 ; n=19$ for cytochalasin $D ; n=18$ for nocodazole). Time zero corresponds to the lowest intensity value measured inside the cell nucleus, before NEP. (h) Time lag between cyclin B1 and tubulin nuclear translocation in DMSO-treated, non-confined cells (black), cells with different treatments (green), and confined cells in the same conditions (magenta).

\section{Figure 3}

(a) Representative images of the nucleus on interphase (left panel) and mitotic (right panels) RPE-1 cells stained with Lamin $A / C$ and DAPI. Note the 
irregularities on the NE surface in interphase cells. Scale bars, $10 \mu \mathrm{m}$. (b) Nuclear irregularity index (NII) in interphase (black) and mitotic cells (green; $\left.{ }^{* *} \mathrm{p}<0.001\right)$. (c) Distribution of NII values for interphase (black) and mitotic cells (green). (d) Representative immunofluorescence images of RPE-1 parental cells in nonconfined, interphase (control interphase; $n=32$ ), non-confined, mitotic (control mitosis; $n=28$ ) and confined interphase (interphase confiner; , $n=19$ ) conditions, stained with cPLA2, DAPI and Lamin A/C. (e) cPLA2 fluorescence intensity ratio between the NE and the nucleoplasm in control interphase cells (black), control mitotic cells (green; ${ }^{* * *} \mathrm{p}<0.001$ ) and confiner interphase cells (magenta; $\left.{ }^{* * *} p<0.001\right)$. (f) Normalized cyclin B1 fluorescence intensity inside the nucleus over time, in cells treated with cPLA2 inhibitor (AACOCF3) with (green; $n=12$ ) or without (black; $n=15)$ confinement $\left({ }^{* *} p<0.01\right)$. (g) Normalized cyclin B1 fluorescence intensity inside the nucleus over time, in cells treated with $\mathrm{Ca}^{2+}$ release inhibitors BAPTA-AM+2APB with (green; $n=11$ ) or without (black; $n=17$ ) confinement $\left({ }^{* *} p<0.001\right)$. Time zero corresponds to the lowest intensity value measured inside the cell nucleus, before NEP. (h) RPE-1 cell expressing cyclinB1-Venus/tubulin-mRFP dividing on an FBN-coated substrate treated with AACOCF3 (cPLA2 inhibitor) without (top panel) and with confinement (lower panel). Time is in min:sec and scale bar corresponds to $10 \mu \mathrm{m}$. Images were acquired with 20 sec interval and time zero corresponds to NEB. (i) Time lag between cyclin B1 and tubulin nuclear translocation in cells treated with AACOCF3 or BAPTA-AM + 2APB in non-confined (black) or confined conditions (green; $\left.{ }^{* * *} p<0.001\right)$.

\section{Figure 4}


(a) RPE-1 cell expressing cyclinB1-Venus/tubulin-mRFP, dividing on a $5 \mathrm{kPa}$ hydrogel (top panel; $n=16$ ) or on glass (lower panel; $n=17$ ). Time is in min:sec and time zero corresponds to NEP. Images were acquired with a $20 \mathrm{sec}$ interval. Scale bar corresponds to $10 \mu \mathrm{m}$. (b) Normalized cyclin B1 fluorescence intensity inside the nucleus over time, in cells seeded on glass (black) or on a $5 \mathrm{kPa}$ hydrogel (green; $\left.{ }^{\star \star *} \mathrm{p}<0.001\right)$. Time zero corresponds to the lowest intensity value measured inside the cell nucleus, before NEP. (c) Time lag between cyclin B1 and tubulin nuclear translocation in cells seeded on glass (black) or cells seeded on a $5 \mathrm{kPa}$ hydrogel (green; $\left.{ }^{* *} \mathrm{p}<0.001\right)$.

\section{Figure 5}

(a) RPE-1 cell expressing H2B-GFP/tubulin-mRFP dividing on an FBN-coated substrate. Time is in min:sec and time zero corresponds to NEP. Images were acquired with a 2 min interval. Scale bar corresponds to $10 \mu \mathrm{m}$. (b) RPE-1 cell expressing H2B-GFP/tubulin-mRFP dividing on an FBN-coated substrate and under a short period of confinement $(n=31)$. Time is in min:sec and time zero corresponds to NEP. Images were acquired with a 2 min interval. Scale bar corresponds to $10 \mu \mathrm{m}$. Centrosomes angle at the time of NEP, in cells dividing without (c) or with (d) confinement. (e) Percentage of mitotic errors in control, non-confined cells, in comparison with cells under a short period of confinement $\left({ }^{*} \mathrm{p}<0.05\right)$. (f) Mitotic timings (NEP-metaphase and NEP-anaphase) in control, non-confined cells, in comparison with cells under a short period of confinement ( $\left.{ }^{*} \mathrm{p}<0.05\right)$. (g) RPE-1 cell expressing H2B-GFP/tubulin-mRFP dividing on a FBN coated substrate in controls (top panel, $n=12$; laser surgery was performed on the cytoplasm) and cells in which NE rupture was induced by laser microsurgery 
(lower panel; $n=15)$. Time is in min:sec and time zero corresponds to NEP. Images were acquired with a 2 min interval. Scale bar corresponds to $10 \mu \mathrm{m}$. (h) Percentage of mitotic errors in control cells and in surgery-treated cells $\left({ }^{* *} p=0.02\right)$. (i) Mitotic timings (NEP-metaphase and NEP-anaphase) in control cells (black) and cells in which NE rupture was induced by laser microsurgery (green; $\left.{ }^{*} \mathrm{p}<0.05 ;{ }^{* *} \mathrm{p}<0.01\right)$.

\section{Supplementary Figure 1}

(a) RPE-1 cell expressing Lap2 $\beta$-mRFP under confinement $(n=29)$. Please note the unfolding of the NE upon confinement. Time is in min:sec. Images were acquired with a $20 \mathrm{sec}$ interval. Scale bar corresponds to $10 \mu \mathrm{m}$. (b) Representative images of RPE- 1 cells stained with TPR (magenta) and an NPC mix (green), acquired with CH-STED, in non-confined (control; $n=44$ ) and confined conditions $(n=55)$. Scale bars correspond to $10 \mu \mathrm{m}$. (c) Quantification of the distance between nuclear pores complexes (NPCs) in control, non-confined

cells (black) and confined cells (green; ${ }^{* *} p<0.001$ ), using our custom-designed MATLAB script.

\section{Supplementary Figure 2}

RPE-1 cells expressing cyclinB1-Venus/tubulin-mRFP dividing on an FBNcoated substrate treated with importazole in non-confined (a) and confined (b) conditions. Time frame in min:sec. Images were acquired with a $20 \mathrm{sec}$ interval. Scale bar corresponds to $10 \mu \mathrm{m}$.

\section{Supplementary Figure 3}


RPE-1 cells expressing cyclinB1-Venus/tubulin-mRFP dividing on an FBNcoated substrate in non-confined conditions, treated with p-nitro-blebbistatin (a; $\mathrm{n}=17$ ) or ML-7 (b; MLCK inhibitor; $\mathrm{n}=13)$. Time in min:sec and time zero corresponds to NEP. Images were acquired with a $20 \mathrm{sec}$ interval. Scale bar corresponds to $10 \mu \mathrm{m}$. (c) Normalized cyclin B1 fluorescence intensity inside the nucleus over time, in control (DMSO; black) and ML7-treated cells (green; $\left.{ }^{* * *} \mathrm{p}<0.001\right)$. Time zero corresponds to the lowest intensity value measured inside the cell nucleus, before NEP. RPE-1 cells expressing cyclinB1-Venus/tubulinmRFP dividing on a FBN coated substrate in non-confined conditions, treated with Y-27632 (d; ROCK inhibitor; $n=23)$, cytochalasin D (e; actin inhibitor; $n=27$ ) and nocodazole (f; microtubule depolymerizer; $\mathrm{n}=20)$. Time is in min:sec and time zero corresponds to NEP. Images were acquired with a $20 \mathrm{sec}$ interval. Scale bar corresponds to $10 \mu \mathrm{m}$.

\section{Supplementary Figure 4}

(a) RPE-1 cell expressing cyclinB1-Venus/tubulin-mRFP dividing on an FBNcoated substrate, treated with BAPTA-AM+2APB in non-confined conditions (top panel) and confined conditions (lower panel). Time in min:sec and time zero corresponds to NEP. Images were acquired with a $20 \mathrm{sec}$ interval. Scale bar corresponds to $10 \mu \mathrm{m}$. (b) Representative images of parental RPE-1 cells stained with TPR (magenta) and an NPC mix (green), acquired with CH-STED in nonconfined (control; $n=62)$ and confined conditions $(n=59)$. In all conditions, cells were treated with p-nitro-blebbistatin. Scale bars corresponds to $10 \mu \mathrm{m}$. (c) Distance between nuclear pore complexes (NPCs) in control non-confined cells 
(black) and confined cells (green; $\left.{ }^{* *} p<0.001\right)$. Quantifications were done using our custom-designed MATLAB script.

\section{Supplementary Figure 5}

(a) RPE-1 cell expressing H2B-GFP/tubulin-mRFP dividing on an FBN-coated substrate, treated with STLC (Eg5 inhibitor), without (top panel) or with confinement (lower panel; $\mathrm{n}=26$ ). Time in min:sec and time zero corresponds to NEP. Images were acquired with a $20 \mathrm{sec}$ interval. Scale bar corresponds to $10 \mu \mathrm{m}$. (b) Quantification of pole-to-pole distance in STLC-treated, non-confined cells (black) and STLC-treated, confined cells (green). (c) Representative image of a western blot to confirm the depletion of DHC with RNAi. Vinculin was used as loading control. (d) RPE-1 cell expressing H2B-GFP/tubulin-mRFP dividing on an FBN-coated substrate treated with DHC RNAi, without (top panel; $n=19$ ) and with confinement (lower panel; $n=19$ ). Time in min:sec and time zero corresponds to NEP. Images were acquired with a $20 \mathrm{sec}$ interval. Scale bar corresponds to $10 \mu \mathrm{m}$. (e) Polar plot showing the distribution of angles between centrosomes and long nuclear axis at the moment of NEP, in cells treated with DHC RNAi, with or without confinement.

\section{References:}

1. Lancaster, O. et al. Mitotic Rounding Alters Cell Geometry to Ensure Efficient Bipolar Spindle Formation. Dev. Cell 25, 270-283 (2013).

2. Cattin, C. J. et al. Mechanical control of mitotic progression in single animal cells. Proc. Natl. Acad. Sci. U. S. A. 112, 1502029112- (2015).

3. Gavet, O. \& Pines, J. Progressive Activation of CyclinB1-Cdk1 
Coordinates Entry to Mitosis. Dev. Cell (2010).

doi:10.1016/j.devcel.2010.02.013

4. Dantas, M., Lima, J. T. \& Ferreira, J. G. Nucleus-Cytoskeleton Crosstalk During Mitotic Entry. 9, 1-9 (2021).

5. Vitiello, E. et al. Acto-myosin force organization modulates centriole separation and PLK4 recruitment to ensure centriole fidelity. Nat.

Commun. (2019). doi:10.1038/s41467-018-07965-6

6. Uroz, M. et al. Regulation of cell cycle progression by cell-cell and cellmatrix forces. Nat. Cell Biol. 1 (2018). doi:10.1038/s41556-018-0107-2

7. Lindqvist, A., Rodríguez-Bravo, V. \& Medema, R. H. The decision to enter mitosis: feedback and redundancy in the mitotic entry network. Journal of Cell Biology (2009). doi:10.1083/jcb.200812045

8. Hagting, A., Karlsson, C., Clute, P., Jackman, M. \& Pines, J. MPF localization is controlled by nuclear export. EMBO J. (1998). doi:10.1093/emboj/17.14.4127

9. Toyoshima, F., Moriguchi, T., Wada, A., Fukuda, M. \& Nishida, E. Nuclear export of cyclin B1 and its possible role in the DNA damage-induced G2 checkpoint. EMBO J. (1998). doi:10.1093/emboj/17.10.2728

10. Pines, J. \& Hunter, T. Isolation of a human cyclin cDNA: Evidence for cyclin mRNA and protein regulation in the cell cycle and for interaction with p34cdc2. Cell (1989). doi:10.1016/0092-8674(89)90936-7

11. Hagting, A., Jackman, M., Simpson, K. \& Pines, J. Translocation of cyclin B1 to the nucleus at prophase requires a phosphorylation-dependent nuclear import signal. Curr. Biol. (1999). doi:10.1016/S09609822(99)80308-X 
12. Li, J., Meyer, A. N. \& Donoghue, D. J. Nuclear localization of cyclin B1 mediates its biological activity and is regulated by phosphorylation. Proc.

Natl. Acad. Sci. U. S. A. (1997). doi:10.1073/pnas.94.2.502

13. Champion, L., Linder, M. I. \& Kutay, U. Cellular Reorganization during Mitotic Entry. Trends in Cell Biology (2017). doi:10.1016/j.tcb.2016.07.004

14. Abe, S. et al. The initial phase of chromosome condensation requires Cdk1-mediated phosphorylation of the CAP-D3 subunit of condensin II. Genes Dev. (2011). doi:10.1101/gad.2016411

15. Huang, S., Chen, C. S. \& Ingber, D. E. Control of cyclin D1, p27(Kip1), and cell cycle progression in human capillary endothelial cells by cell shape and cytoskeletal tension. Mol Biol Cell (1998).

16. Gudipaty, S. A. et al. Mechanical stretch triggers rapid epithelial cell division through Piezo1. Nature (2017). doi:10.1038/nature21407

17. Benham-Pyle, B. W., Pruitt, B. L. \& Nelson, W. J. Mechanical strain induces E-cadherin-dependent Yap1 and $\beta$-catenin activation to drive cell cycle entry. Science (80-. ). (2015). doi:10.1126/science.aaa4559

18. Klein, E. A. et al. Cell-Cycle Control by Physiological Matrix Elasticity and In Vivo Tissue Stiffening. Curr. Biol. (2009). doi:10.1016/j.cub.2009.07.069

19. Aureille, J. et al. Nuclear envelope deformation controls cell cycle progression in response to mechanical force. EMBO Rep. (2019). doi:10.15252/embr.201948084

20. Vianay, B. et al. Variation in traction forces during cell cycle progression. Biol. Cell 110, 91-96 (2018).

21. Lombardi, M. L. \& Lammerding, J. Keeping the LINC: The importance of 
nucleocytoskeletal coupling in intracellular force transmission and cellular function. in Biochemical Society Transactions (2011).

doi:10.1042/BST20110686

22. Arsenovic, P. T. et al. Nesprin-2G, a Component of the Nuclear LINC Complex, Is Subject to Myosin-Dependent Tension. Biophys. J. (2016). doi:10.1016/j.bpj.2015.11.014

23. Elosegui-Artola, A. et al. Force Triggers YAP Nuclear Entry by Regulating Transport across Nuclear Pores. Cell (2017).

doi:10.1016/j.cell.2017.10.008

24. Lomakin, A. J. et al. The nucleus acts as a ruler tailoring cell responses to spatial constraints. Science (80-. ). (2020). doi:10.1126/science.aba2894

25. Jacchetti, E. et al. The nuclear import of the transcription factor MyoD is reduced in mesenchymal stem cells grown in a 3D micro-engineered niche. Sci. Rep. (2021). doi:10.1038/s41598-021-81920-2

26. Nava, M. M. et al. Heterochromatin-Driven Nuclear Softening Protects the Genome against Mechanical Stress-Induced Damage. Cell (2020). doi:10.1016/j.cell.2020.03.052

27. Swift, J. et al. Nuclear lamin-A scales with tissue stiffness and enhances matrix-directed differentiation. Science (80-. ). (2013).

doi:10.1126/science. 1240104

28. Venturini, V. et al. The nucleus measures shape changes for cellular proprioception to control dynamic cell behavior. Science (80-. ). (2020). doi:10.1126/science.aba2644

29. Nunes, V. et al. Centrosome-nuclear axis repositioning drives the assembly of a bipolar spindle scaffold to ensure mitotic fidelity. Mol. Biol. 
Cell (2020). doi:10.1091/mbc.e20-01-0047

30. Chen, C. S. Geometric Control of Cell Life and Death. Science (80-. ). (1997). doi:10.1126/science.276.5317.1425

31. Peter, M., Nakagawa, J., Dorée, M., Labbé, J. C. \& Nigg, E. A. In vitro disassembly of the nuclear lamina and $\mathrm{M}$ phase-specific phosphorylation of lamins by cdc2 kinase. Cell (1990). doi:10.1016/0092-8674(90)90471-

$\mathrm{P}$

32. Heald, R. \& McKeon, F. Mutations of phosphorylation sites in lamin A that prevent nuclear lamina disassembly in mitosis. Cell (1990). doi:10.1016/0092-8674(90)90470-Y

33. Moore, J. D., Yang, J., Truant, R. \& Kornbluth, S. Nuclear import of Cdk/cyclin complexes: Identification of distinct mechanisms for import of Cdk2/cyclin E and Cdc2/cyclin B1. J. Cell Biol. (1999).

doi:10.1083/jcb.144.2.213

34. Enyedi, B., Jelcic, M. \& Niethammer, P. The Cell Nucleus Serves as a Mechanotransducer of Tissue Damage-Induced Inflammation. Cell 165, $1160-1170$ (2016).

35. Strauss, B. et al. Cyclin B1 is essential for mitosis in mouse embryos, and its nuclear export sets the time for mitosis. J. Cell Biol. (2018). doi:10.1083/jcb.201612147

36. Furuno, N., Elzen, N. Den \& Pines, J. Human cyclin A is required for mitosis until mid prophase. J. Cell Biol. (1999). doi:10.1083/jcb.147.2.295

37. Tse, H. T. K., Weaver, W. M. C. \& Carlo, D. Increased asymmetric and multi-daughter cell division in mechanically confined microenvironments. PLoS One (2012). doi:10.1371/journal.pone.0038986 
38. Silkworth, W. T., Nardi, I. K., Paul, R., Mogilner, A. \& Cimini, D. Timing of centrosome separation is important for accurate chromosome segregation. Mol. Biol. Cell (2012). doi:10.1091/mbc.E11-02-0095

39. Le Berre, M., Zlotek-Zlotkiewicz, E., Bonazzi, D., Lautenschlaeger, F. \& Piel, M. Methods for two-dimensional cell confinement. Methods Cell Biol. 121, 213-229 (2014).

40. Schindelin, J. et al. Fiji: An open-source platform for biological-image analysis. Nature Methods (2012). doi:10.1038/nmeth.2019 


\section{bioRxiv preprint doi: https://doi.org/10.1101/2021.09.23.461473; this version posted September 23 2021. The copyright holder for this license to display the preprint in}
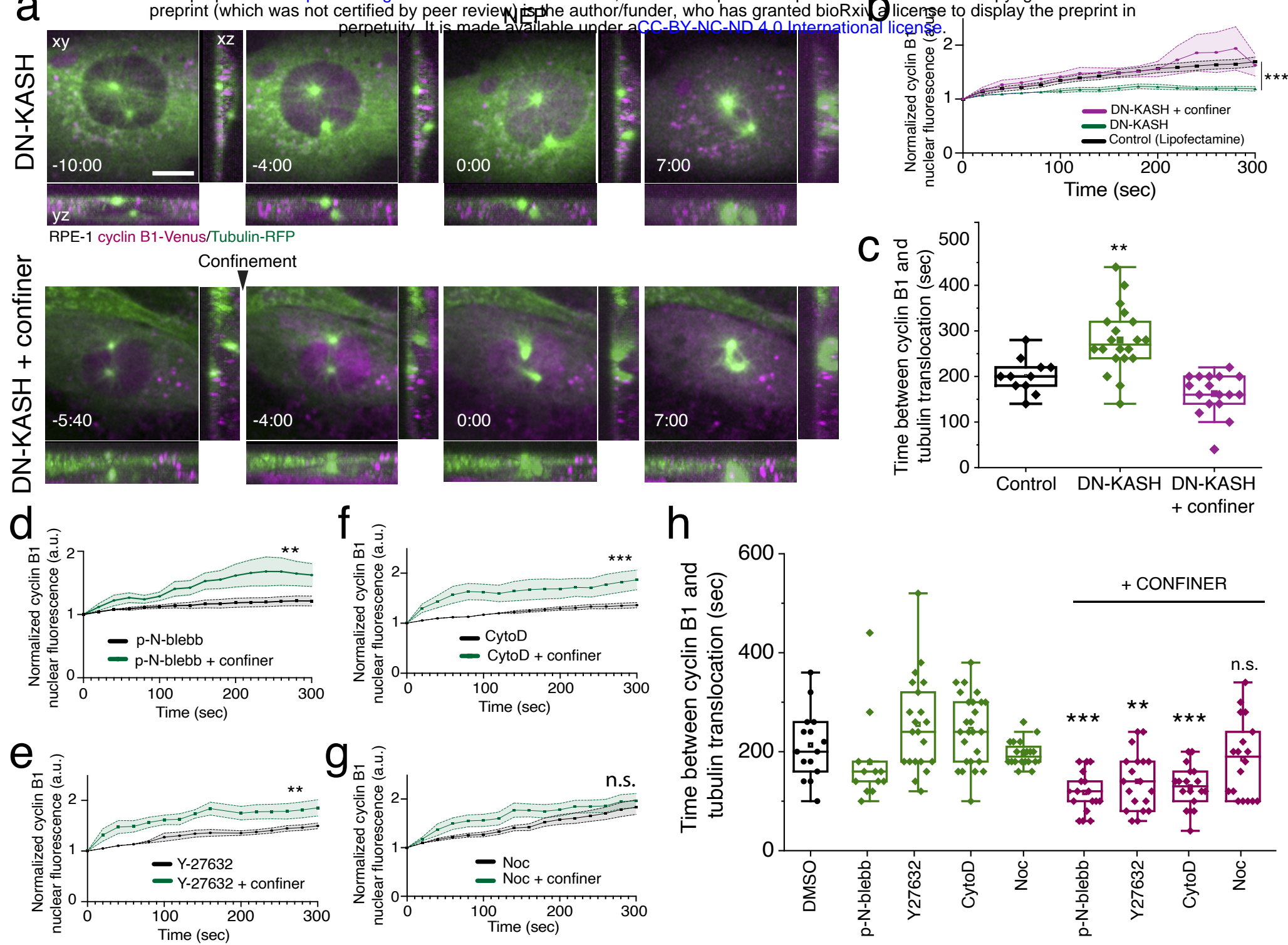

Figure 2 
bioRxiv preprint doi: https://doi.org/10.1101/21.09.23.461473; this version posted September 23,021. The copyright holder for this

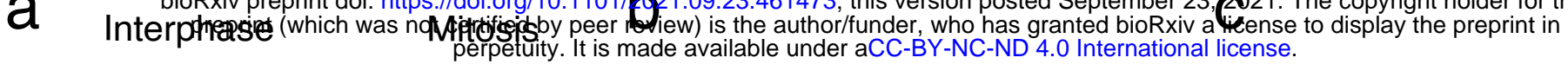
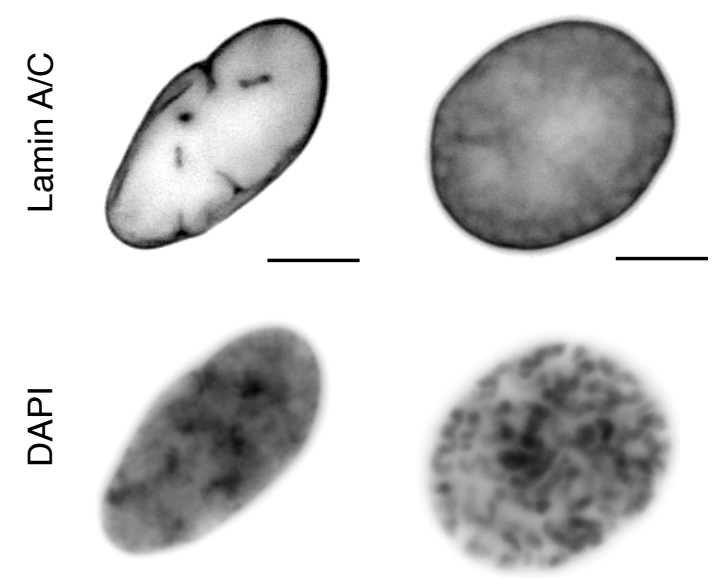

d

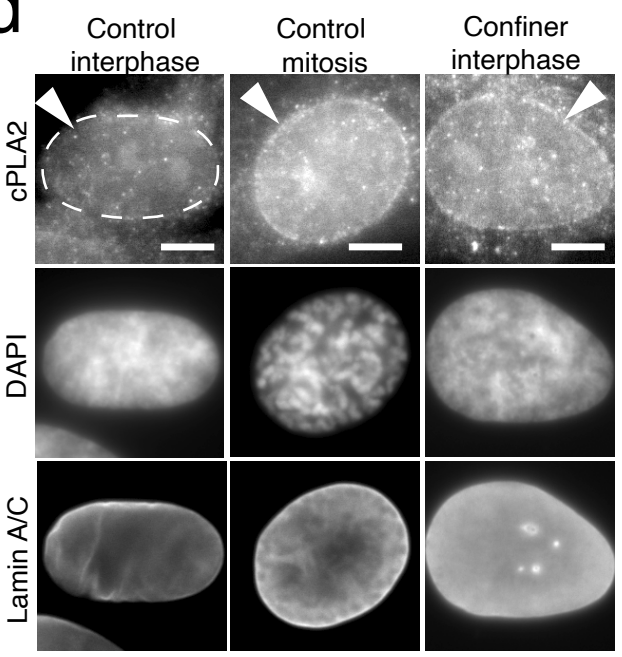

RPE-1 parental
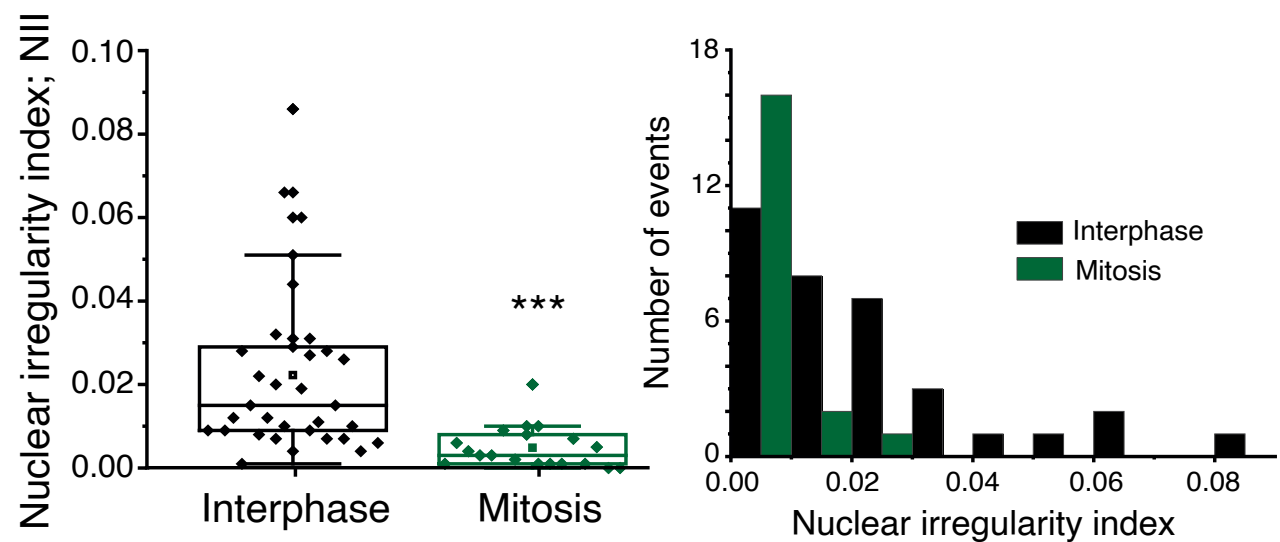

f

e

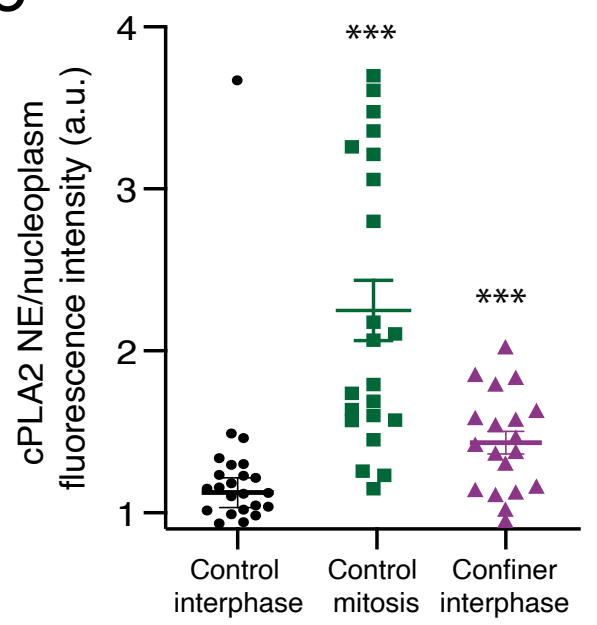

9
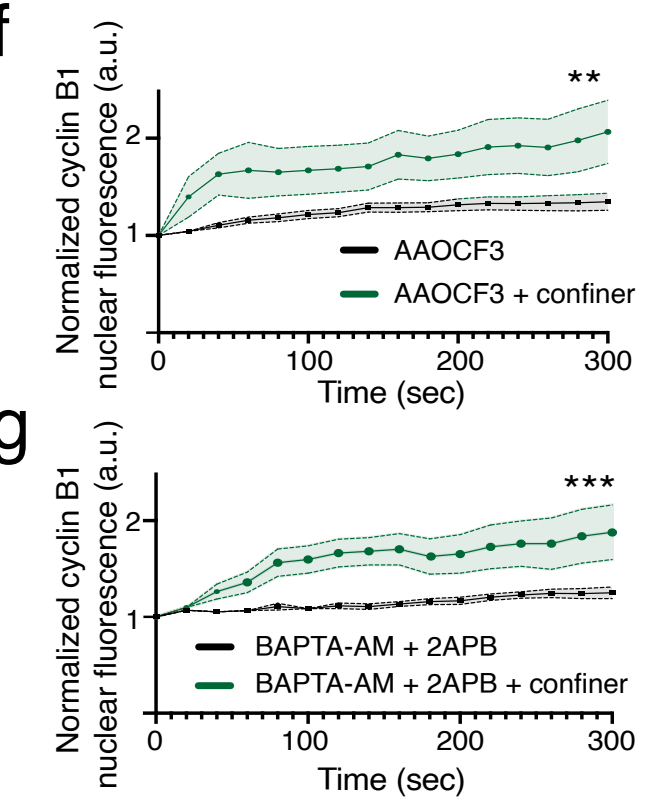

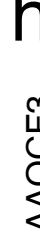

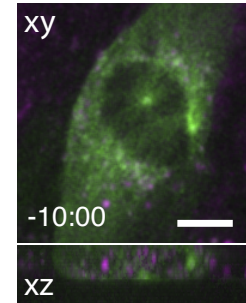

RPE-1 cyclin B1-Venus/Tubulin-RFP

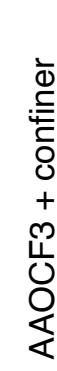

Confinement

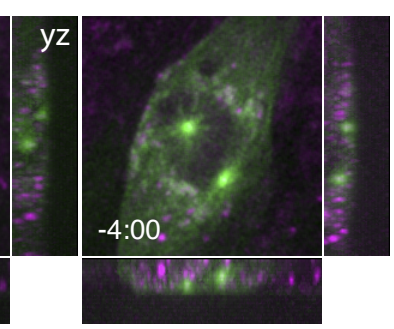

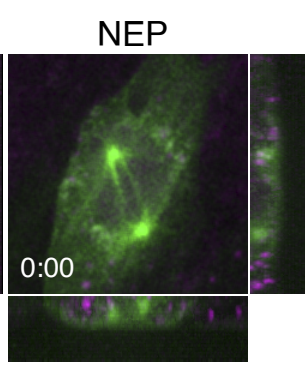
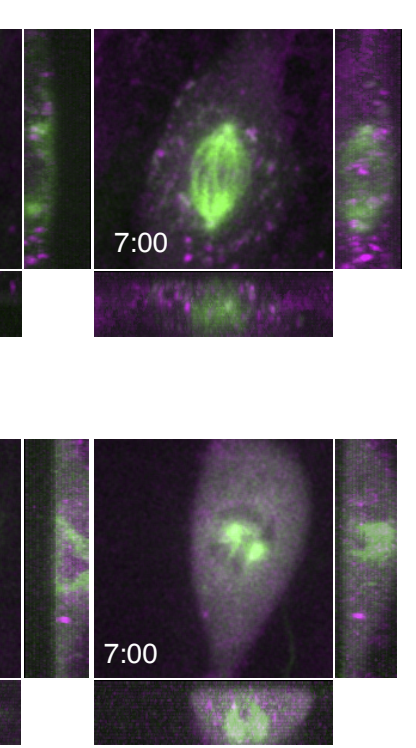

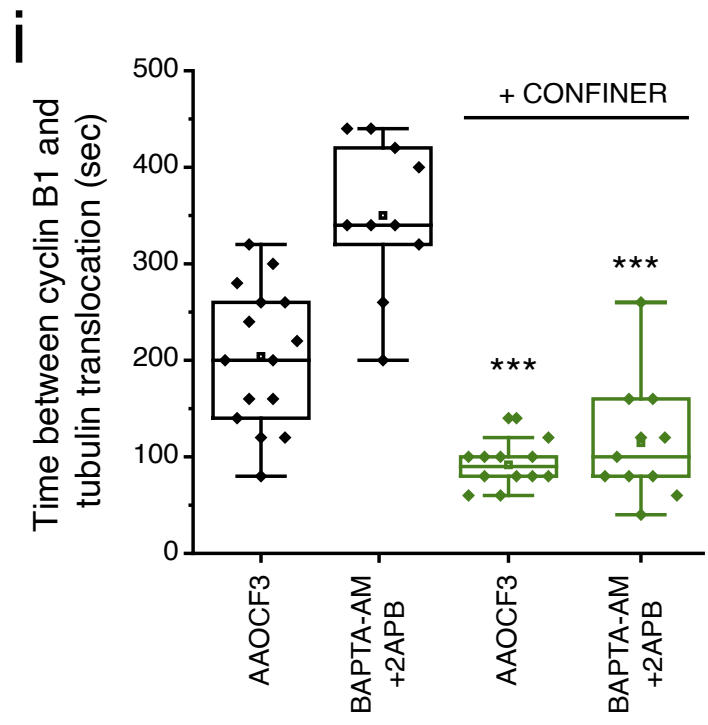


a

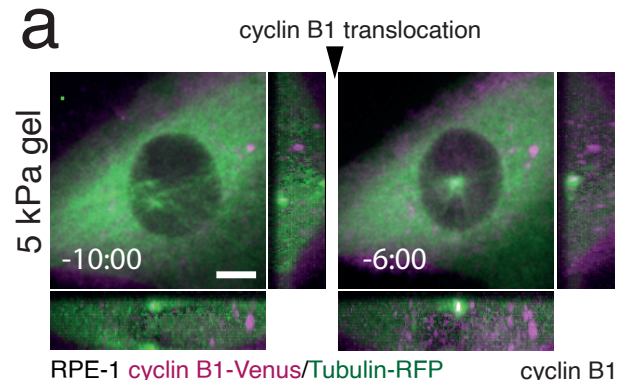

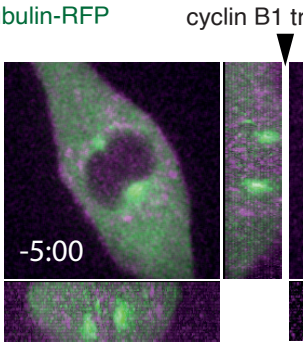

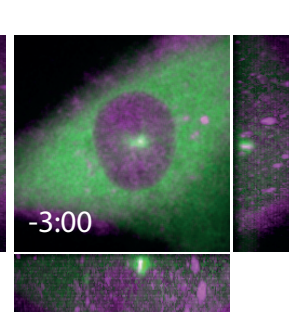

translocation

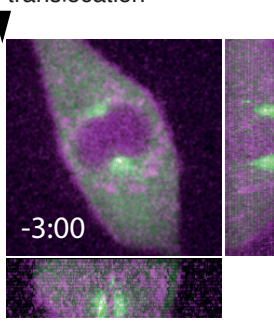

NEP
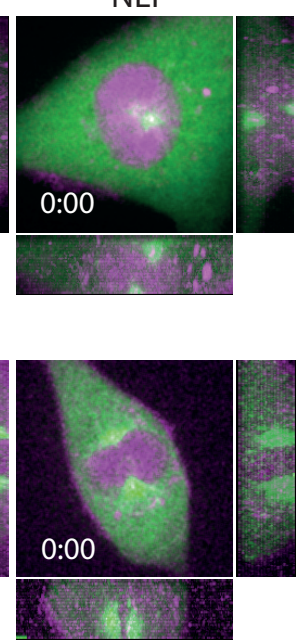

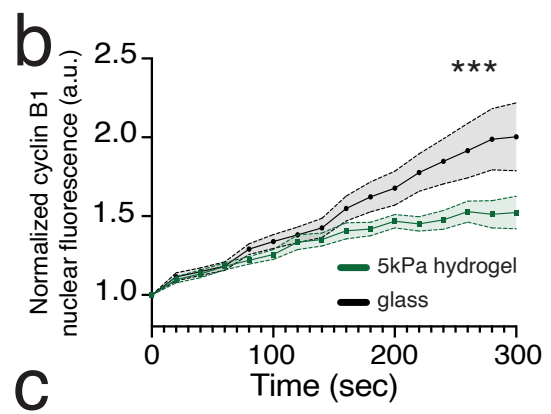

C
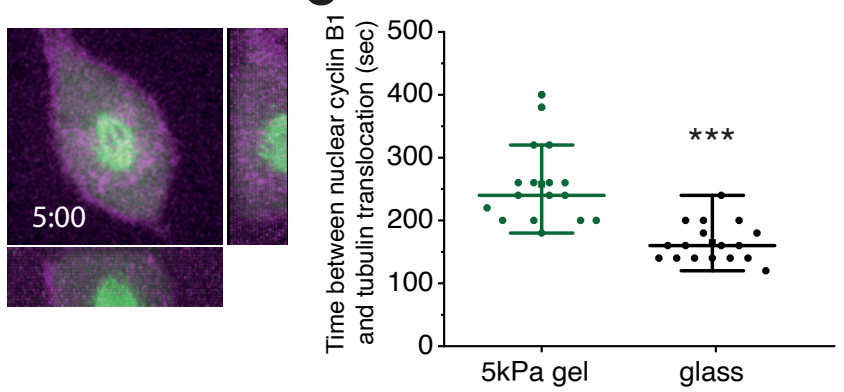

Figure 4 

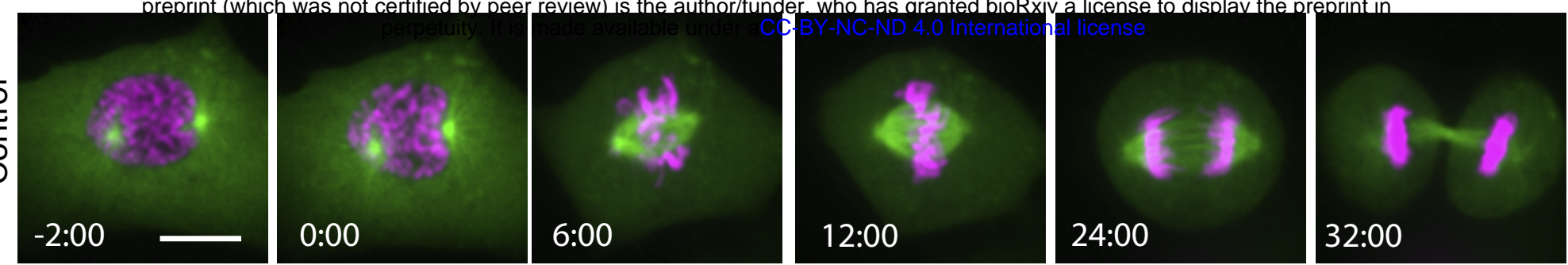

RPE-1 H2B-GFP/Tubulin-RFP

\section{confinement}

(0:00) b
$\stackrel{\frac{1}{0}}{\frac{1}{c}}$

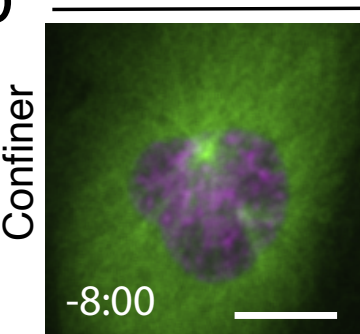

C

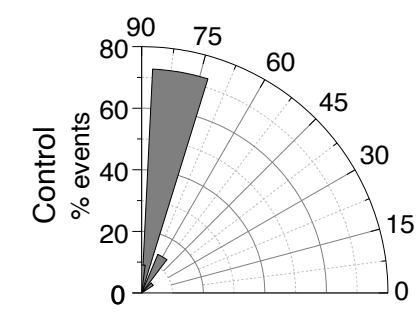

7 NEP

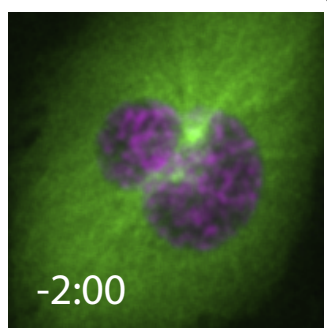

d

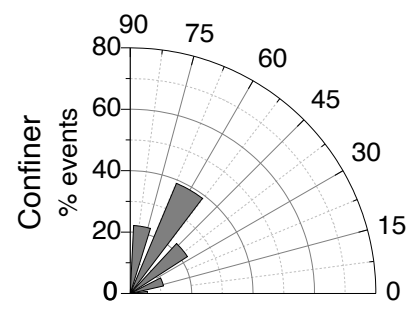

release

$(4: 00)$

$\nabla$
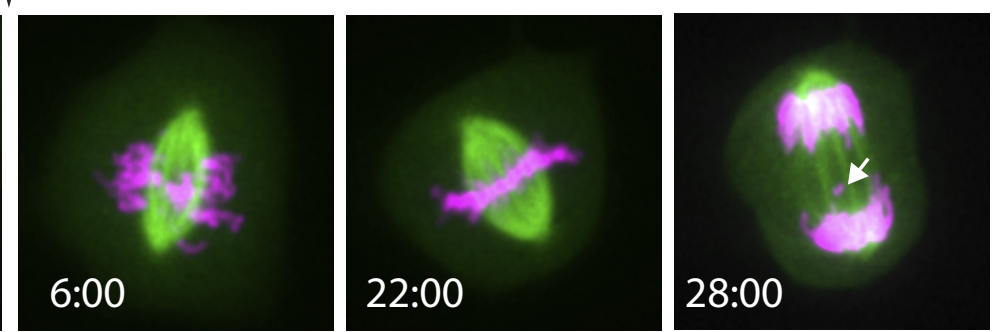

e

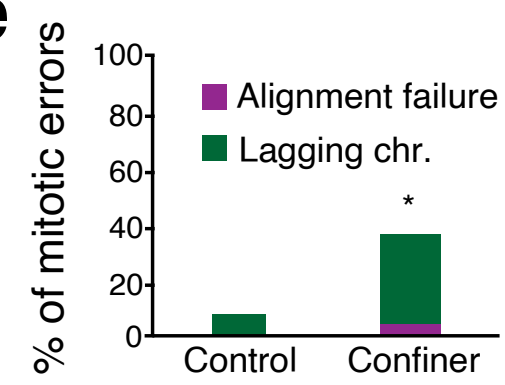

$f$

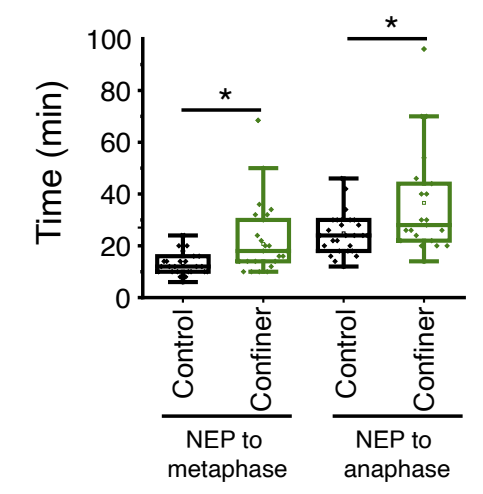

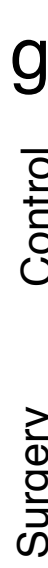

Before surgery

용

응

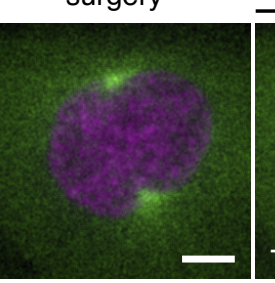

$-6: 00$

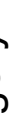

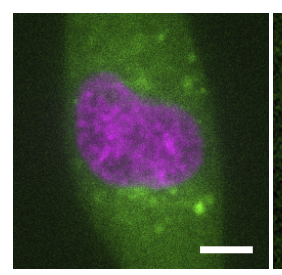

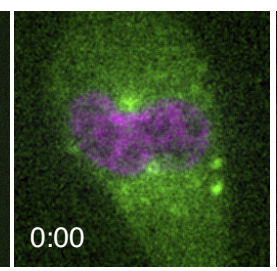

After surgery

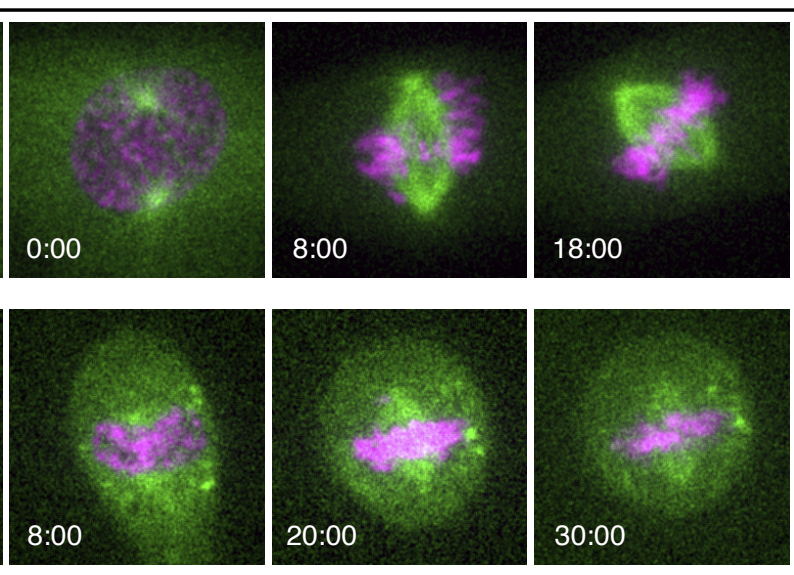

$40: 00$

\section{h}
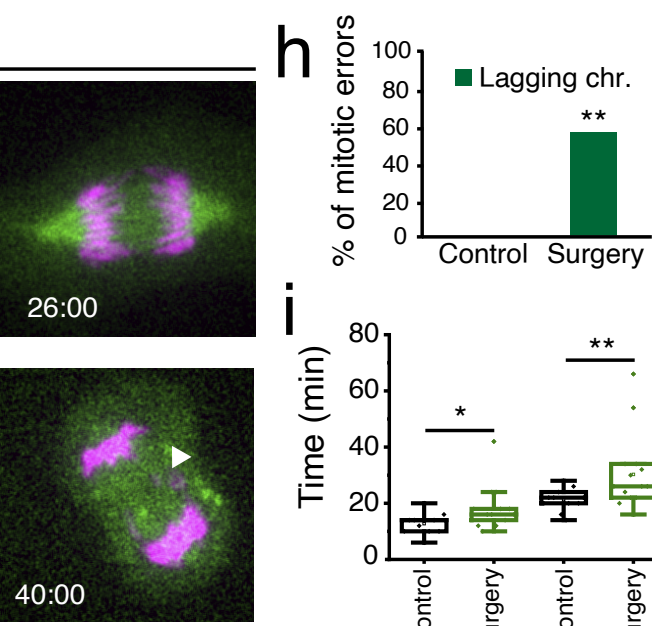

\section{Figure 5}


bioRxiv preprint doi: $h t t p s / / d o i . o r g / 10.1101 / 2021.09 .23 .461473$; this version posted September 23, 2021. The copyright holder for this areprint (which was not certified by peer review) is the attinfirfenderntwho has granted bioRxiv a license to display the preprint in a perpetuity. It is made available under-ACC-BY-NC-ND 4.0 International license.

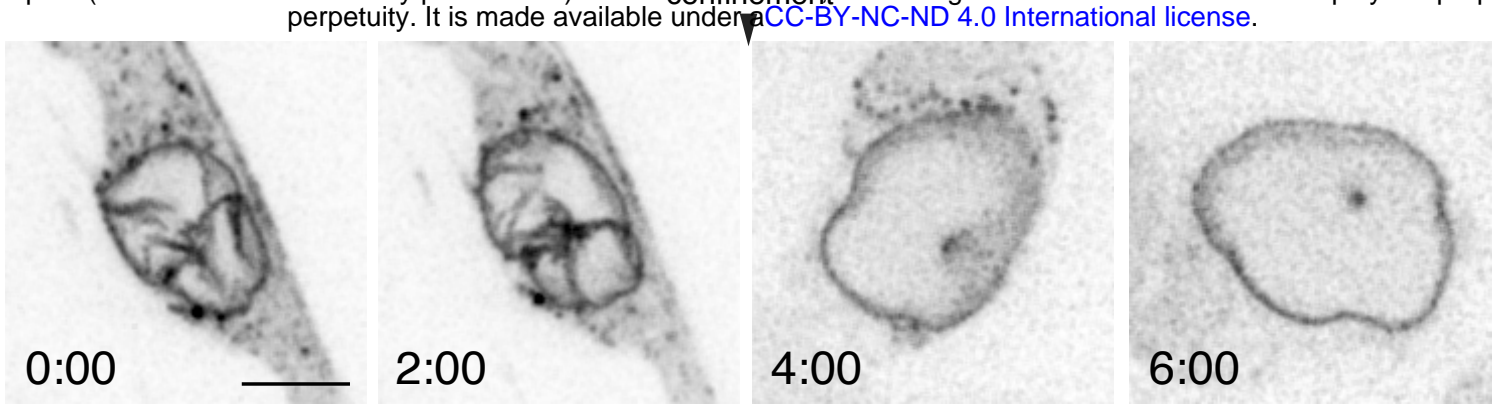

RPE-1 Lap2 $\beta-m R F P$

b

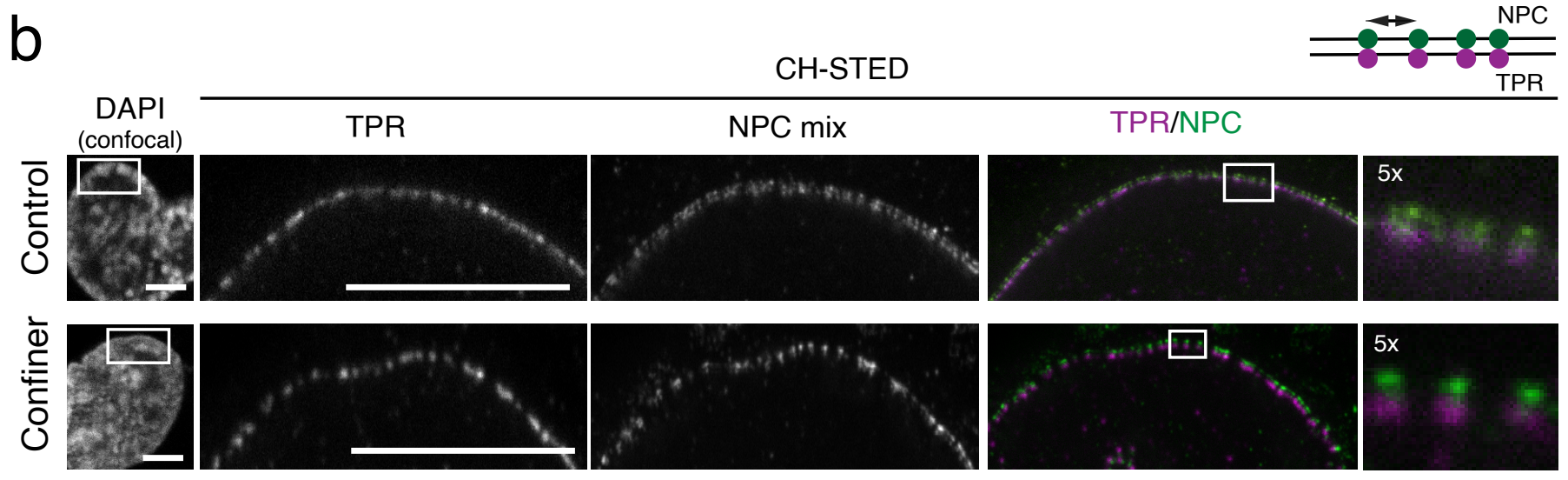

RPE-1 parental

C

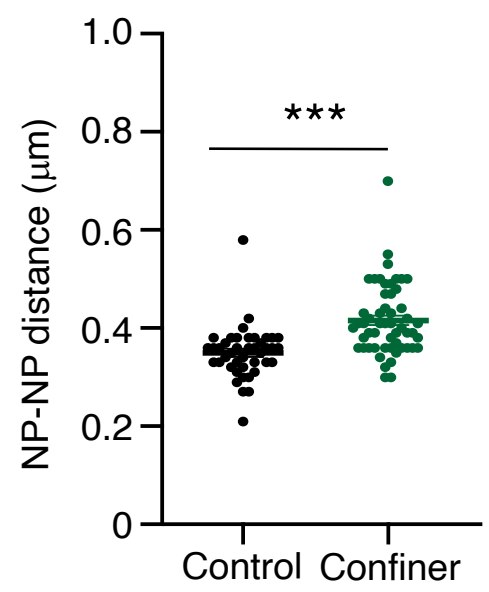

Supplementary Figure 1 
bioRxiv preprint doi: https://doi.org/10.1101/2021.09.23.461473. this version posted September 23, 2021. The copyright holder for this preprint (which was not certified by peer review) is the author/funder, who has granted bioRxiv a license to display the preprint in

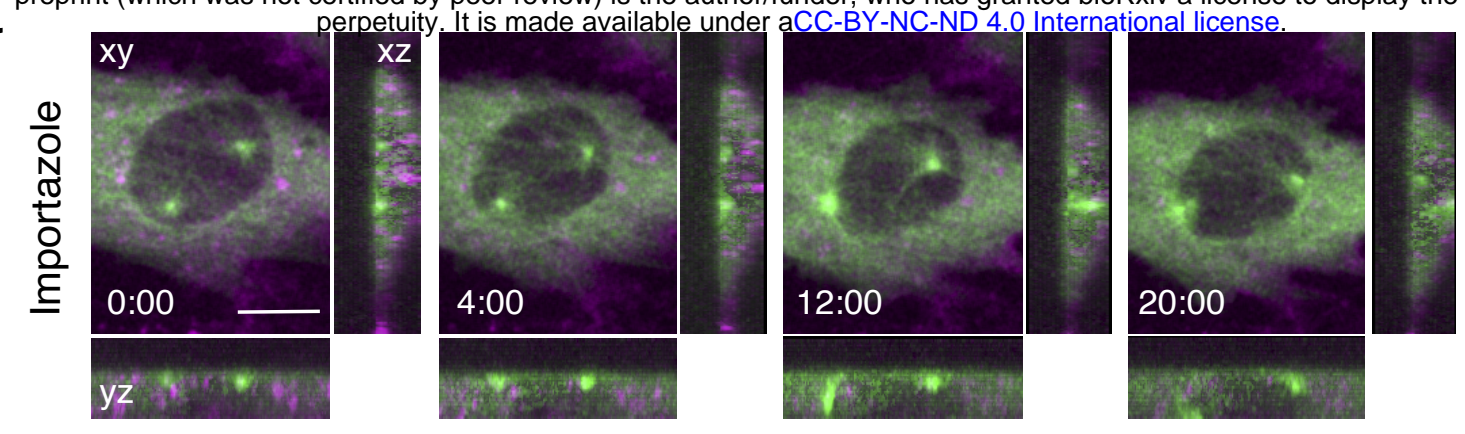

RPE-1 cyclin B1-Venus/Tubulin-RFP

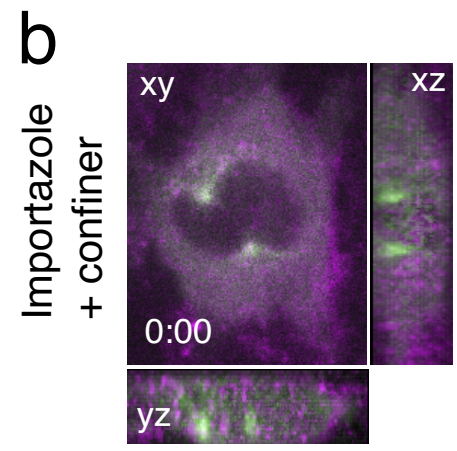

confinement
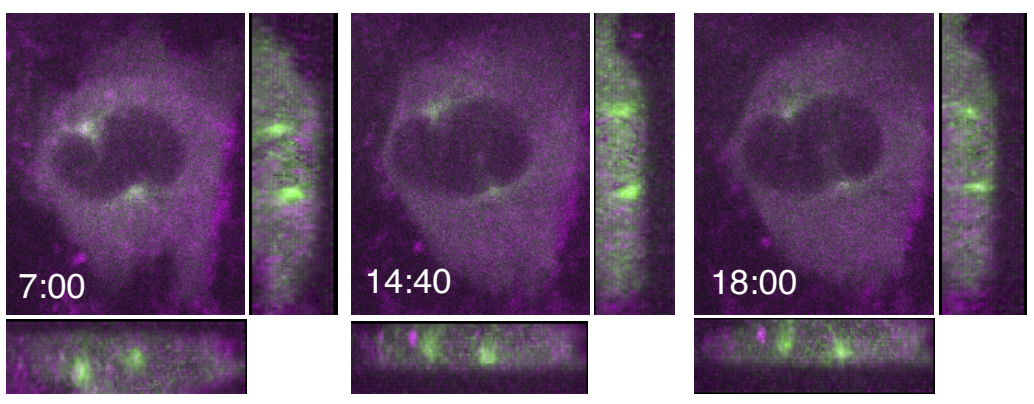

\section{Supplementary Figure 2}


bioRxiv preprint doi: https://doi.org/10.1101/2021.09.23.461473; this version posted September 23, 2021. The copyright holder for this preprint (which was not certified by peer review) is the author/funder, who has granted bioRxiv a license to display the preprint in

a perpetuity. It is made available under aCC-BY-NC-ND 4.0 International license.

NEP
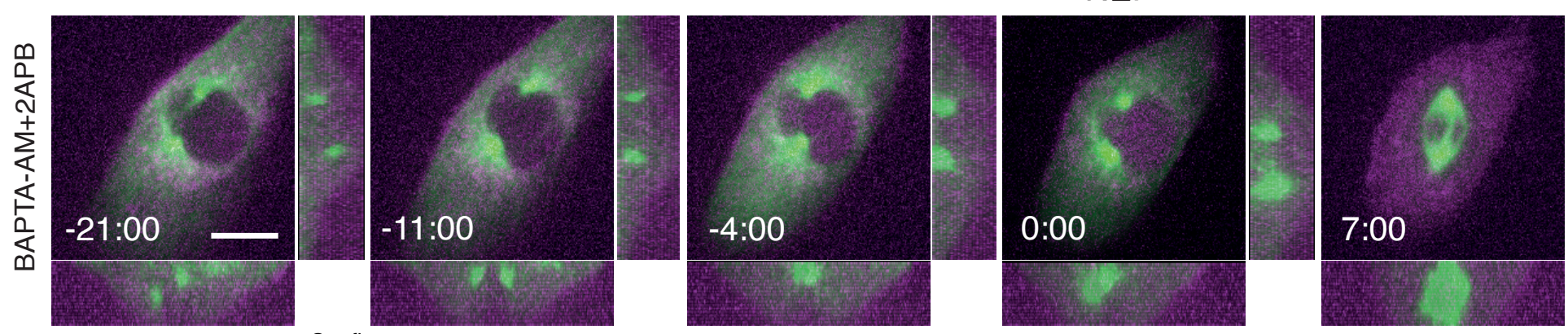

Confinement
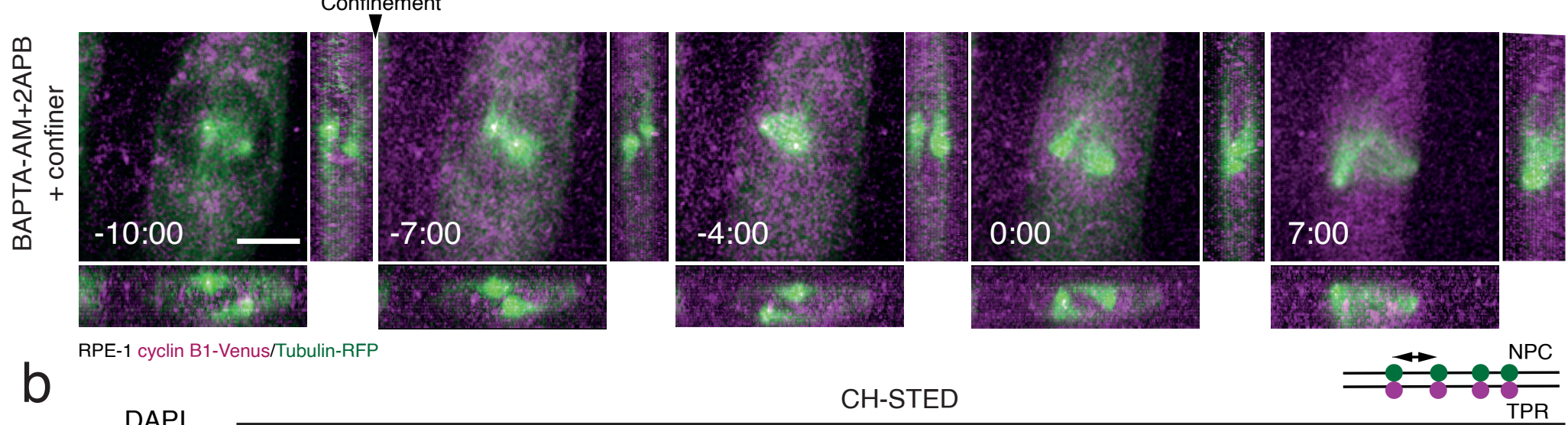

b

RPE-1 cyclin B1-Venus/Tubulin-RFP

CH-STED
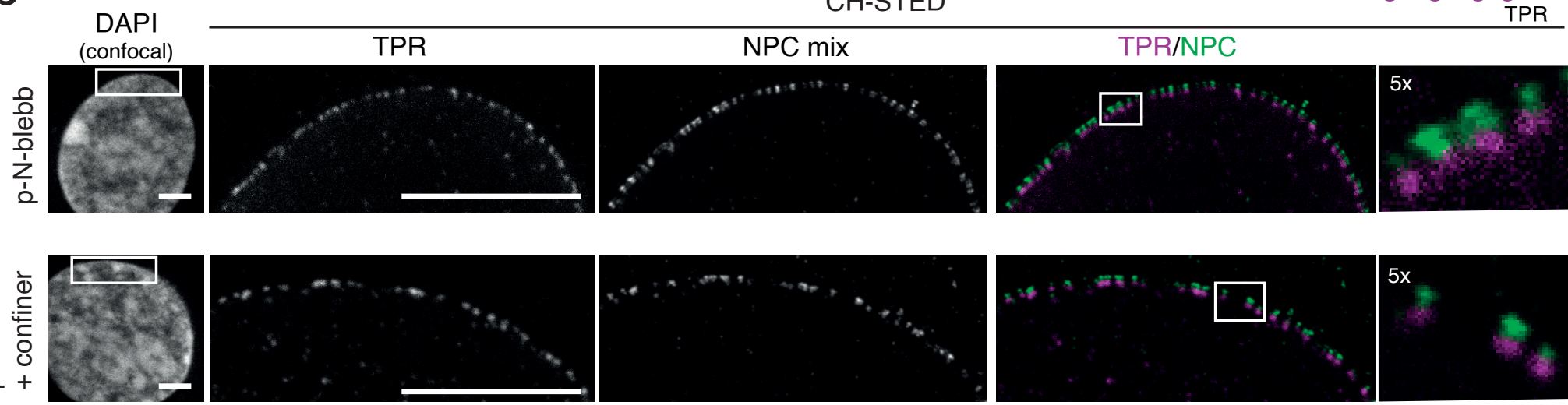

RPE-1parental
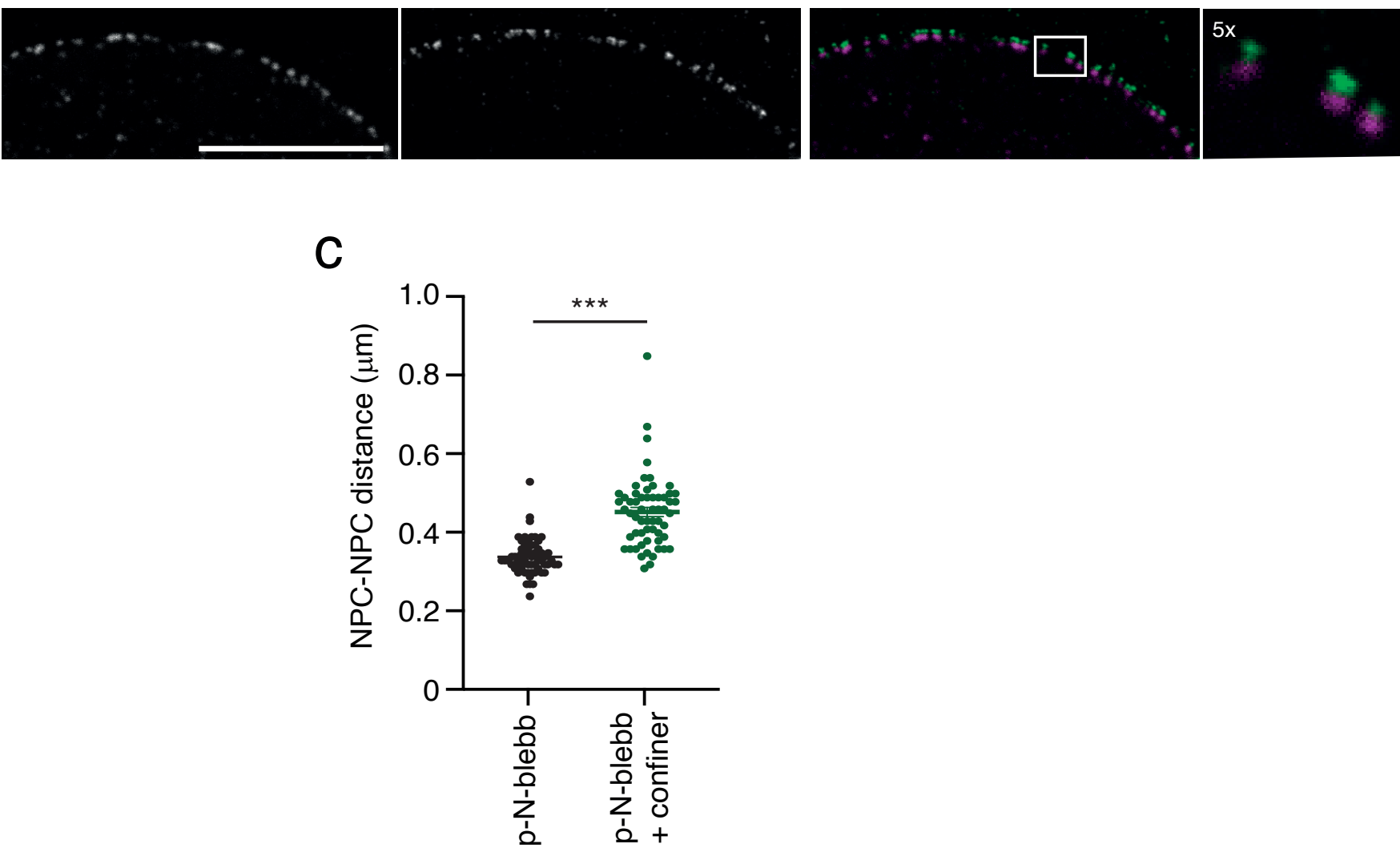

Supplementary Figure 4 
a

bioRxiv preprint doi: https://doi.org/10.1101/2021.09.23.461473; this version posted September 23, 2021. The copyright, holder for this preprint (which was not certified by peer review) is the author/funder, who has granted bioRxiv a license
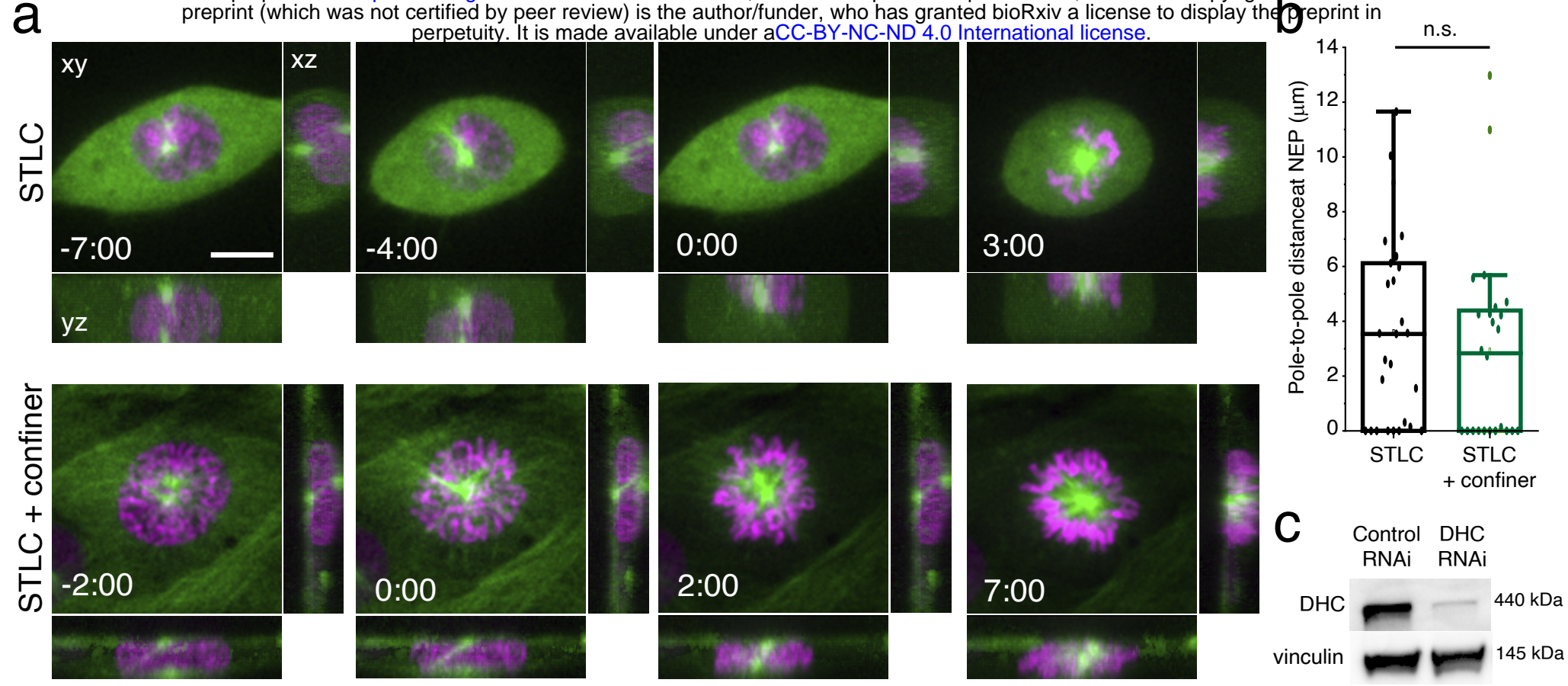

d

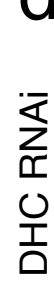

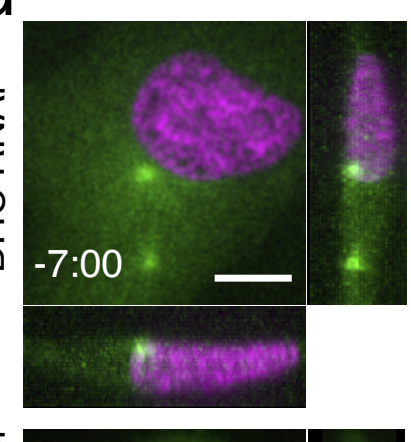

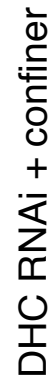
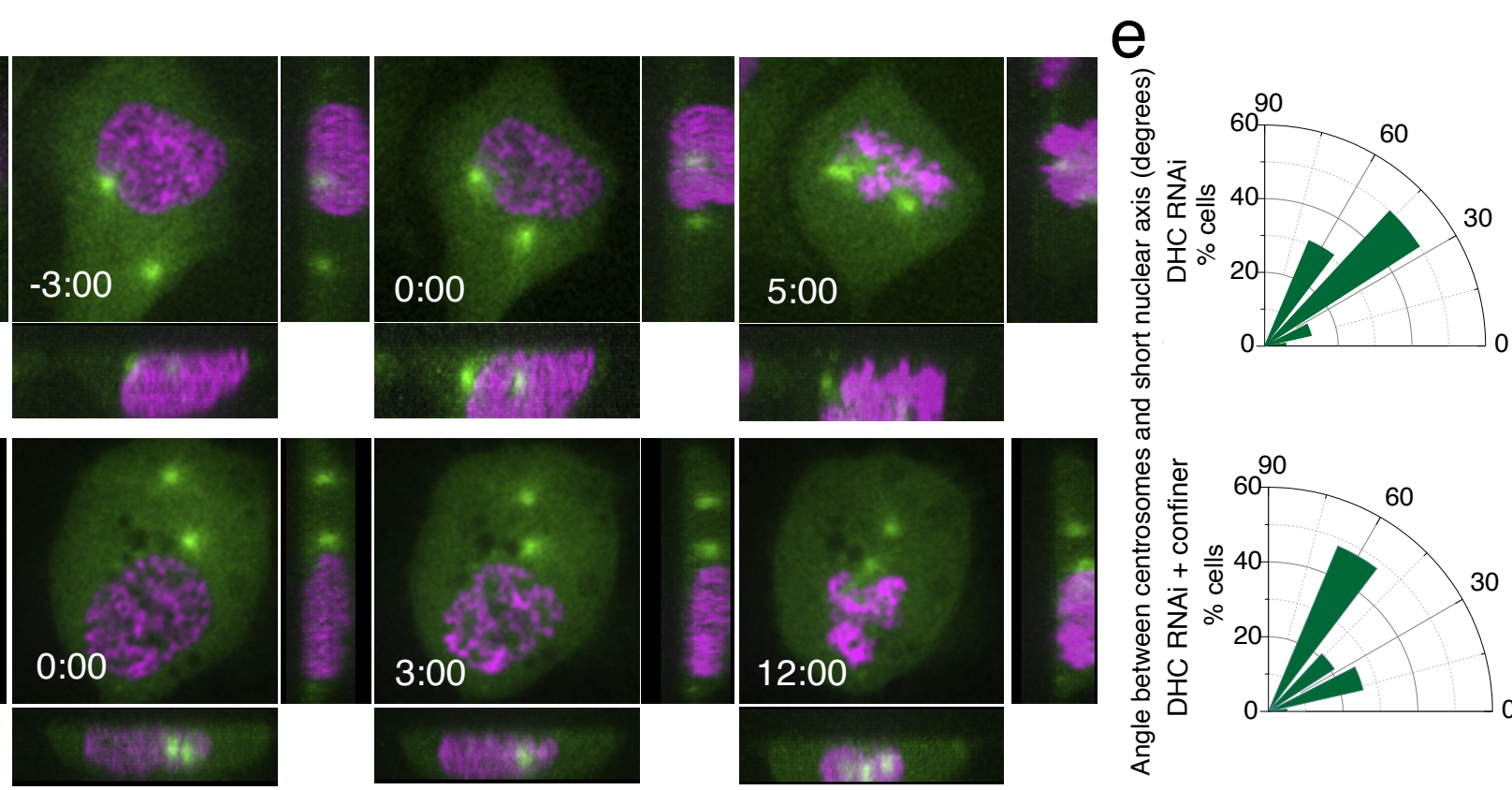

\section{Supplementary Figure 5}

